Natural Hazards and Earth System Sciences, 5, 799-819, 2005

SRef-ID: 1684-9981/nhess/2005-5-799

European Geosciences Union

(c) 2005 Author(s). This work is licensed

under a Creative Commons License.

\title{
Modelling debris flows down general channels
}

\author{
S. P. Pudasaini, Y. Wang, and K. Hutter \\ Department of Mechanics - AG III, Darmstadt University of Technology, Hochschulstrasse 1, 64289, Germany
}

Received: 2 June 2005 - Revised: 8 August 2005 - Accepted: 9 September 2005 - Published: 26 October 2005

\begin{abstract}
This paper is an extension of the single-phase cohesionless dry granular avalanche model over curved and twisted channels proposed by Pudasaini and Hutter (2003). It is a generalisation of the Savage and Hutter $(1989,1991)$ equations based on simple channel topography to a twophase fluid-solid mixture of debris material. Important terms emerging from the correct treatment of the kinematic and dynamic boundary condition, and the variable basal topography are systematically taken into account. For vanishing fluid contribution and torsion-free channel topography our new model equations exactly degenerate to the previous Savage-Hutter model equations while such a degeneration was not possible by the Iverson and Denlinger (2001) model, which, in fact, also aimed to extend the Savage and Hutter model. The model equations of this paper have been rigorously derived; they include the effects of the curvature and torsion of the topography, generally for arbitrarily curved and twisted channels of variable channel width. The equations are put into a standard conservative form of partial differential equations. From these one can easily infer the importance and influence of the pore-fluid-pressure distribution in debris flow dynamics. The solid-phase is modelled by applying a Coulomb dry friction law whereas the fluid phase is assumed to be an incompressible Newtonian fluid. Input parameters of the equations are the internal and bed friction angles of the solid particles, the viscosity and volume fraction of the fluid, the total mixture density and the pore pressure distribution of the fluid at the bed. Given the bed topography and initial geometry and the initial velocity profile of the debris mixture, the model equations are able to describe the dynamics of the depth profile and bed parallel depth-averaged velocity distribution from the initial position to the final deposit. A shock capturing, total variation diminishing numerical scheme is implemented to solve the highly non-linear equations. Simulation results present the combined effects of curvature, torsion and pore pressure
\end{abstract}

Correspondence to: S. P. Pudasaini

(pudasain@mechanik.tu-darmstadt.de) on the dynamics of the flow over a general basal topography. These simulation results reveal new physical insight of debris flows over such non-trivial topography. Model equations are applied to laboratory avalanche and debris-flow-flume tests. Very good agreement between the theory and experiments is established.

\section{Introduction}

Debris and mud flows are multiphase, gravity driven flows consisting of randomly dispersed interacting phases. In the geophysical context they consist of solid components with different grain size and shape, of liquid and possibly air. A theory accounting for all these interactions is still out of reach, so that most of the present models mainly focus attention to limiting cases: $(i)$ single phase dry cohesionless granular continuum of a body consisting of particles of a nominal mean, representative size, and (ii) saturated binary mixture consisting of a solid constituent and a fluid that fills the entire pore space.

Model (i) predicts the avalanching flow of dry granular materials. Its most popular version seems to be the class of Savage-Hutter type models (Savage and Hutter, 1989, 1991; Gray et al., 1999; Pudasaini and Hutter, 2003; Pudasaini et al., 2003, 2005a). It is based on the assumption that the rapidly moving granular mass is density preserving and the internal, material and basal sliding properties can be described by cohesionless Mohr-Coulomb type frictional laws involving as material parameters the internal and bed friction angles. Supposing predominance of sliding over shearing a depth averaging is meaningful without loosing great accuracy of the dynamical description as given by the emerging equations. These equations have been applied to different catastrophic flow configurations (see, e.g., Zwinger et al., 2003; Pitman et al., 2003; Patra et al., 2003). Laboratory and some field experiments have corroborated their validity for catastrophic land slide and avalanche events (see, e.g., Gray et al., 1999; Denlinger and Iverson, 2001; Pudasaini, 2003; 
Iverson et al., 2004; and Pudasaini et al., 2005b). Their limits of applicability are discussed in Hutter et al. (2005).

Many debris and mud flow events are triggered by heavy rain falls, so that the motion of the sliding mass of debris or mud is better described by a mixture of a solid and a fluid phase under conditions of saturation. This is indeed the underlying concept of all debris flow models known to us that go beyond a single phase description. The most prominent examples are the debris flow models of Hungr (1995), Iverson (1997), Iverson and Denlinger (2001), Iverson et al. (2004), Denlinger and Iverson $(2001,2004)$ and Pitman and Le (2005), but pioneering work of Takahashi (1991) should also be mentioned.

The underlying simplifications are performed on two different levels. First, it is assumed that the mixture density can be taken as constant. Moreover, the seepage velocity is introduced as a variable that replaces the fluid velocity as a basic field. From the mass and momentum balance of the mixture as a whole and the implementation that the seepage velocity is negligibly small, it then follows that the velocity of the solid constituent is the only remaining kinematic field ${ }^{1}$. The momentum equation, formulated for the mixture as a whole, contains then as constitutive quantities the solid and fluid partial stresses which, respectively, are modelled as a Coulomb material, just as in the Savage-Hutter theory and as a Newtonian fluid with constant viscosity. So, formally, this reduced two phase debris model appears as if it were a one-constituent model with a fluid stress composed of a pressure and a dissipative stress. A Darcy type interaction force does not enter.

The second simplifying assumption is based on the shallow geometry of the debris masses. It motivates introduction of the thickness averaging to arrive at model equations for the depth and depth-integrated velocities tangential to the basal surface. In this process the total traction of the solid and fluid constituents perpendicular to the free surface must be divided into solid and fluid constituents, and this is done, as in Iverson (1997) and Iverson and Denlinger (2001), by introducing a factor $\Lambda_{f}$ such that the normal stress $\left(1-\Lambda_{f}\right) T_{(z z)}$ and $\Lambda_{f} T_{(z z)}$ is composed of the partial solids and fluids stresses. $\Lambda_{f}$ is treated phenomenologically as an internal variable that may follow from a diffusion equation for the fluid pressure. The emerging equations are then so structured that the limit $\Lambda_{f}=0$ recovers the Pudasaini and Hutter (2003) model, whilst the limit $\Lambda_{f}=1$ generates the purely viscous equations appropriate for a slush or a debris avalanche. The former constitute a purely hyperbolic, the latter a mixed hyperbolic-parabolic system of equations. These equations contain a scale dependent dimensionless quantity $N_{R}$ (see Iverson, 1997; Iverson and Denlinger, 2001) which is the fluid volume fraction weighted Reynolds number.

\footnotetext{
${ }^{1}$ The fluid velocity is set equal to the solid velocity whenever their difference arises; this appears formally to be tantamount to the neglection of the Darcy interaction force, but it manifests itself through the formulation of the fluid constituent Cauchy stress.
}

The physical foundation of this debris flow model is based on the recognition that the fluid stress significantly contributes to the dynamics of the flow. In the soil mechanics context, where the viscous properties of the fluid are ordinarily ignored, this manifests itself as the significant role played by the pore pressure; here the viscous contribution is added, and it stabilises the numerics because the viscous stresses and the diffusion equation for the pressure introduce parabolicity into the system. Mathematically, the present formulation adopts an orthogonal coordinate system along a curved and twisted master curve (see Pudasaini and Hutter, 2003) that is suggested by the thalweg of the valley or corrie, through which the debris flow takes place. This is advantageous and preferable to the horizontal and vertical coordinates used by others (see, e.g., Hungr, 1995; Iverson and Denlinger, 2001, 2004; Zwinger et al., 2003; Pitman et al., 2003; Patra et al., 2005), because it is more naturally adjusted to the geometries which one often encounters in debris flows - and it is more accurate whenever slopes are large $\left(30^{\circ}-50^{\circ}\right)$ and the mountain valley is generally curved and twisted. In fact, a mathematically correct asymptotic analysis cannot rigorously be performed if the coordinates are not following the topography.

The model equations include the effects of the curvature and torsion of the topography in the dynamics of the debris flow and influence of the pore fluid pressure distribution is made explicit. Important terms emerging from the correct treatment of the kinematic and dynamic boundary condition, and the variable basal topography are systematically taken into account. For vanishing fluid contribution and torsion free bed topography our new model equations exactly degenerate to the previous model equations of Savage and Hutter $(1989,1991)$; this was not possible with the Iverson and Denlinger (2001) model, which in fact, aimed to generalise the Savage-Hutter model. A shock capturing, total variation diminishing numerical scheme is implemented to solve the highly non-linear model equations. Simulation results present the combined effects of curvature, torsion and porefluid pressure on the dynamics of the debris flow over varied topography. These simulation results reveal the physics of the debris flows over such non-trivial topography which could not be achieved with previous model equations.

The model equations are examined by comparing their numerical results with two different experiments (Denlinger and Iverson, 2001): (i) Small-scale laboratory avalanche of dry sand sliding down an inclined rectangular flume that merges continuously to the horizontal deposition zone; (ii) Large-scale water-saturated debris flows in an out-door flume. The former case deals with the dynamics of deformation of avalanche from initiation to deposit, whilst the latter is concerned with the debris flow surge development and its hydrographs at different cross-sectional positions of the flume. Very good agreement between theory and experiments is observed.

In what follows, we shall present in Sect. 2 the governing field equations and boundary conditions. In Sect. 3 the general coordinate system is presented and Sect. 4 repeats 
the model equations of Pudasaini and Hutter (2003) as an extension of the one-constituent Savage-Hutter model; these equations are preparatory, but equally necessary for the formulation of the general model. Section 5 then addresses the peculiarities related to the fluid components. The final, depth integrated equations are summarised in Sect. 6, and the new features of the model are discussed in Sect. 7. Section 8 briefly introduces the numerical method and Sect. 9 discusses results obtained for flow configurations through curved and twisted channels and some comparisons of these with flume experiments of Denlinger and Iverson (2001). Section 10 presents a discussion of the achievements and draws inferences for further work.

\section{Field equations for a binary-mixture of a solid and a fluid}

\subsection{Mass and momentum balance equations}

We start with the standard balance equations for binary mixtures. The mass balance equations for the solid and fluid are, respectively,

$$
\frac{\partial\left(\rho_{\alpha} v_{\alpha}\right)}{\partial t}+\nabla \cdot\left(\rho_{\alpha} v_{\alpha} \mathbf{u}_{\alpha}\right)=m_{\alpha}, \quad(\alpha=s, f)
$$

where $s$ and $f$ stand for "solid" and "fluid" and $\nabla$ is the gradient operator, $\partial / \partial t$ indicates partial differentiation with respect to time, $\rho_{s}$ and $\rho_{f}$ are the true mass density of the solid and fluid, $v_{s}$ and $v_{f}$ are the volume fraction of the solid and fluid, respectively. Similarly, $\mathbf{u}_{s}$ and $\mathbf{u}_{f}$ are the solid and fluid velocities, respectively, and $m_{s}$ and $m_{f}$ are the respective rates of solid and fluid mass production, per unit volume. We consider only saturated debris material, so, the volume fractions must obey the saturation condition $v_{s}+v_{f}=1$. We define the total mixture mass density $\rho$ and the barycentric velocity $\mathbf{u}$ as

$\rho=\sum_{\alpha=s, f} \rho_{\alpha} v_{\alpha}, \quad \mathbf{u}=\sum_{\alpha=s, f}\left(\rho_{\alpha} v_{\alpha} \mathbf{u}_{\alpha}\right) / \rho$.

In the sequel we shall assume no mass exchange between the solid and fluid constituents, i.e., $m_{s}=m_{f}=0$ and the constituents are incompressible. So, by dividing (1) by $\rho_{\alpha}$ and adding the resulting equations for $\alpha=s$ and $\alpha=f$ we obtain the mixture mass balance as

$\nabla \cdot v_{f}\left(\mathbf{u}_{f}-\mathbf{u}_{s}\right)+\nabla \cdot \mathbf{u}_{s}=0$.

From the mixture theory, we have the momentum balance equations for the solids and fluid

$$
\begin{aligned}
& \rho_{\alpha} v_{\alpha}\left[\partial \mathbf{u}_{\alpha} / \partial t+\mathbf{u}_{\alpha} \cdot \nabla \mathbf{u}_{\alpha}\right]=\nabla \cdot \mathbf{T}_{\alpha}+\rho_{\alpha} v_{\alpha} \mathbf{g}+\mathbf{f}_{\alpha}, \\
& (\alpha=s, f)
\end{aligned}
$$

where $\mathbf{g}$ is the gravitational acceleration, $\mathbf{T}_{s}$ and $\mathbf{T}_{f}$ are the partial Cauchy stress tensors for the solid and fluid phases, respectively, and $\mathbf{f}_{\alpha}$, with $\mathbf{f}_{s}=-\mathbf{f}_{f}=\mathbf{f}$, is the interaction force per unit volume that results from momentum exchange between the solid and fluid constituents.
In order to simplify the momentum equations we focus on the motion of the solids and analyse the motion of the fluid relative to that of the solids. For this purpose, following the spirit of Iverson (1997), we need to define the relevant fluid velocity which is the fluid specific discharge divided by the fluid volume fraction $\mathbf{q} / v_{f}=\mathbf{u}_{f}-\mathbf{u}_{s}$. Substituting this relation into the fluid momentum equation yields

$\rho_{f} v_{f}\left[\frac{\partial}{\partial t}\left(\frac{\mathbf{q}}{v_{f}}+\mathbf{u}_{s}\right)+\frac{\mathbf{q}}{v_{f}} \cdot \nabla\left(\frac{\mathbf{q}}{v_{f}}+\mathbf{u}_{s}\right)+\mathbf{u}_{s} \cdot \nabla\left(\frac{\mathbf{q}}{v_{f}}+\mathbf{u}_{s}\right)\right]$

$=\nabla \cdot \mathbf{T}_{f}+\rho_{f} v_{f} \mathbf{g}-\mathbf{f}$.

The ensuing analysis is based on the fact that $\left|\mathbf{u}_{s}-\mathbf{u}_{f}\right| \ll\left|\mathbf{u}_{s}\right|$. Iverson (1997) justifies the estimate $\left|\mathbf{q} / v_{f}\right| \ll\left|\mathbf{u}_{s}\right|$. Therefore, Eq. (5) reduces to

$\rho_{f} v_{f}\left[\frac{\partial \mathbf{u}_{s}}{\partial t}+\mathbf{u}_{s} \cdot \nabla \mathbf{u}_{s}\right]=\nabla \cdot \mathbf{T}_{f}+\rho_{f} v_{f} \mathbf{g}-\mathbf{f}$.

Adding (4) for $\alpha=s$ and (6) results in the simplified momentum equation for the solid-fluid mixture

$\rho\left[\frac{\partial \mathbf{u}_{s}}{\partial t}+\mathbf{u}_{s} \cdot \nabla \mathbf{u}_{s}\right]=\nabla \cdot\left(\mathbf{T}_{s}+\mathbf{T}_{f}\right)+\rho \mathbf{g}$.

Similarly, the mass balance Eq. (3) reduces to

$\nabla \cdot \mathbf{u}_{s}=0$

Equations (7) and (8), originally proposed by Iverson (1997), constitute the governing equations for debris flows. As can be seen, there are two main differences between these equations and analogous equations governing the motion of a single-phase granular solid, e.g., Savage and Hutter (1989). These are: $(i)$ they involve the total mixture density $\rho$, and (ii) the influence of the fluid stress $\mathbf{T}_{f}$ is explicitly incorporated into the momentum equation of the mixture. For simplicity, from now on we will write the velocity field as $\mathbf{u}$ instead of $\mathbf{u}_{s}$.

\subsection{Evolution of stresses}

The solid phase is assumed to satisfy a Mohr-Coulomb yield criterion in which the internal shear stress $\mathbf{S}$ and the normal pressure $N$ are related by

$|\mathbf{S}|=N \tan \phi$

where $\phi$ is the internal angle of friction. Alternatively, the fluid stresses obey the conventional linear law that governs the behaviour of incompressible Newtonian fluids, explicitly

$\mathbf{T}_{f}=-p \mathbf{I}+2 v_{f} \mu_{f} \mathbf{D}$

where $p$ is the pressure, I the unit tensor, $\mu_{f}$ the pore fluid viscosity which is multiplied by the fluid volume fraction $v_{f}$ because only this fraction of the mixture produces viscous stresses, and $\mathbf{D}$ is the strain rate tensor given by $\mathbf{D}=\frac{1}{2}\left[\operatorname{grad} \mathbf{u}+(\operatorname{grad} \mathbf{u})^{T}\right]$ 


\subsection{Boundary conditions}

Saturated debris flows possess two distinct surfaces that bound the domain of the moving material, i.e., $(i)$ the free surface, and (ii) the bed. Kinematic and dynamic boundary conditions must be formulated at these surfaces; their complexity depends on the complexity of the theory as well as on the physical processes one intends to include at these boundaries. In general, the free surface is a material surface of the solid, but not for the fluid. If saturated conditions are assumed this implies a surface run-off of water. This run-off must be small and will henceforth be ignored. Similarly, we will also ignore any discharge of water into the ground below the basal surface.

\section{Kinematic boundary conditions.}

The above simplifying assumptions imply the kinematic boundary conditions for the solid phase

$$
\frac{\partial F^{\beta}}{\partial t}+\mathbf{u}^{\beta} \cdot \nabla F^{\beta}=0, \quad(\beta=s, b)
$$

where the superscripts ' $s$ ' and ' $b$ ' indicate that the respective variable is evaluated at the surface, $F^{s}(\mathbf{x}, t)=0$, and the base, $F^{b}(\mathbf{x}, t)=0$, respectively. Equation (11) may also be written as

$$
\begin{aligned}
& \frac{\partial F^{\beta}}{\partial t}+\left.\mathbf{u}\right|_{\beta} \cdot \nabla F^{\beta}=\left|\nabla F^{\beta}\right| a^{\beta}, \quad a^{\beta}=\left(\mathbf{u}^{\beta}-\left.\mathbf{u}\right|_{\beta}\right) \cdot \mathbf{n}^{\beta}, \\
& (\beta=s, b)
\end{aligned}
$$

where $\mathbf{n}^{\beta}=\nabla F^{\beta} /\left|\nabla F^{\beta}\right|$ are the surface and basal outward unit normals and $a^{\beta}$ are accumulation and erosion/deposition functions for $\beta=s$ and $\beta=b$, respectively. They must be parameterised. If the normal component of the velocity of the free-surface, $\mathbf{u}^{s}$, and of basal surface, $\mathbf{u}^{b}$, agree with the normal velocities at the free and basal surfaces, $\left.\mathbf{u}\right|_{s}$ and $\left.\mathbf{u}\right|_{b}$, respectively, then accumulation of solid mass at the free surface and erosion/deposition at the bed are ignored. If $a^{s}=0$, there is no run-off at the free-surface, and if $a^{b}=0$, there is no entrainment at the bed. Below we will limit attention to this case.

\section{Dynamic boundary conditions.}

The free surface of the debris flow is traction free for both constituents while the base satisfies a Coulomb dry-friction sliding law for the solid constituent. That is,

$\mathbf{T}_{s}^{s} \mathbf{n}^{s}=\mathbf{0}, \quad \mathbf{T}_{f}^{s} \mathbf{n}^{s}=\mathbf{0}$,

$\left|\mathbf{S}^{b}\right|=N^{b} \tan \delta, \quad$ or

$\mathbf{T}_{s}^{b} \mathbf{n}^{b}-\mathbf{n}^{b}\left(\mathbf{n}^{b} \cdot \mathbf{T}_{s}^{b} \mathbf{n}^{b}\right)=\left(\mathbf{u}^{b} /\left|\mathbf{u}^{b}\right|\right)\left(\mathbf{n}^{b} \cdot \mathbf{T}_{s}^{b} \mathbf{n}^{b}\right) \tan \delta$,

where $\delta$ is the basal angle of friction. If $a^{s} \neq 0$ and $a^{b} \neq 0$, then Eqs. (13), (14) must be complemented by the impulse contribution due to surface run-off and entrainment of mass from the ground.
The dynamic boundary conditions at the free surface imply that it is stress-free for both constituents, and so the mixture. For the fluid at the base a no-slip condition or a viscous sliding relation can be incorporated. Equations (7) and (8) together with the solid and fluid constitutive relations (9)(10) and boundary conditions (11)-(14) constitute a basis for modelling debris flow dynamics.

\section{Orthogonal general coordinate system}

Curved surfaces strongly influence the flow dynamics because transverse shearing and cross-stream momentum transport occur when the topography obstructs or redirects the motion due to curvature and torsion. Resistance due to basal friction is modified by "centrifugal forces" induced by bed curvature and torsion. The channel topography and the geometry of the debris flow in the lateral and longitudinal directions are illustrated in Fig. 1. Similarly, Fig. 2 represents the geometric description of the coordinate system and some prototype channel geometries with uniform, diverging and converging curved and twisted channels that can be used in the transportation of granular materials. In what follows, except in Sect. 9.4, every quantity in this paper is written in non-dimensional form.

Pudasaini and Hutter (2003) extended the Savage-Hutter theory to flows of dry granular masses in a non-uniformly curved and twisted channel. First, we will outline the geometric configurations they implemented for dry granular flow that we will also adopt in this paper. Consider a debris flowprone landscape and a subregion of it where the topography allows identification of the likely debris flow track. A space curve parallel to the thalweg of the valley is singled out as a master curve $C$ (which can be obtained, e.g., by shifting the thalweg along its normal direction) from which the track topography will be modelled. The curvature and torsion of the master curve, $\kappa=\kappa(x), \tau=\tau(x)$, are either assumed to be known or can be computed from digital elevation Geographic-Information-Systems (GIS) data as functions of the arc length $x$ of the master curve. Then, an orthogonal coordinate system along the master curve is introduced; the model equations are derived in this general coordinate system. In the equations of this paper, $(x, y)$ form a curved reference surface, where $x$ is the coordinate along the thalweg of a mountain valley, while $y$ is the circular arc length in a cross-sectional plane perpendicular to the thalweg of which the value is determined by the relation $y=\varepsilon \theta z_{T}$, where $\varepsilon$ is the aspect ratio between the debris flow height and extent, $\theta$ is the azimuthal angle which accounts for the cross-slope curvature and $z_{T}$ (usually $z_{T} \gg 1$ ) is the radial distance between the master curve and the thalweg and $z$ is the coordinate perpendicular to the reference topography.

The present theory is designed to model the flow of debris over channels having general curvature and torsion. Although there are other models that consider the problem of debris flow motion over curved slopes (e.g., Iverson and Denlinger, 2001; Pitman et al., 2003), the model equations of this 

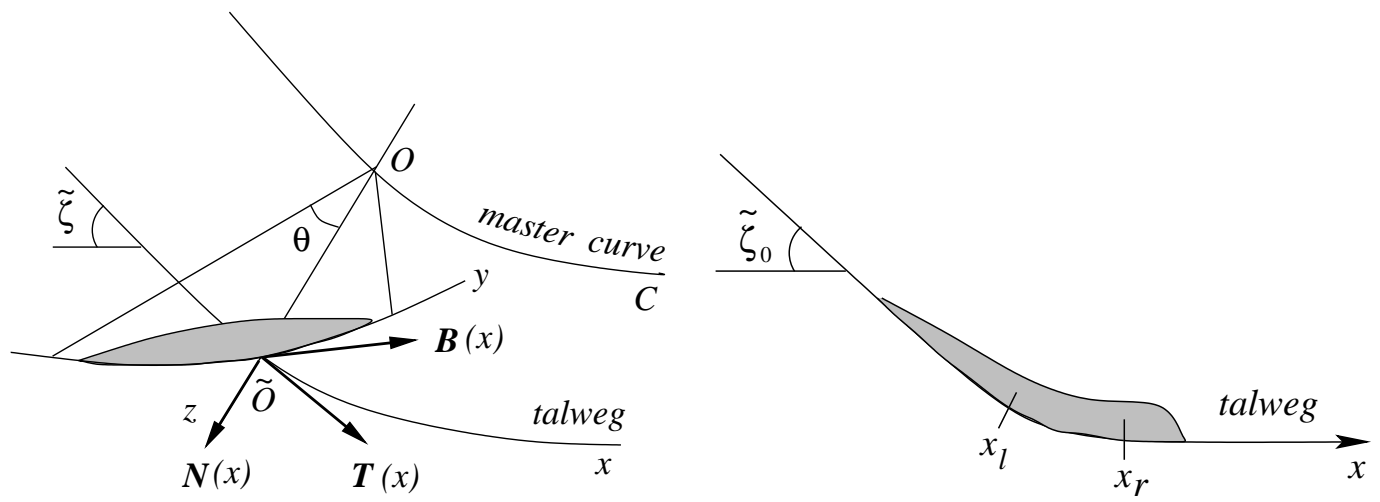

Fig. 1. Left: The debris flow domain in the lateral direction occupies a region in a circular section of a plane perpendicular to the thalweg of the valley and $\theta$ is the azimuthal angle in this plane. $O \tilde{O}=z_{T}$ is the radial distance between the master curve and the thalweg. $\{\mathbf{T}, \mathbf{N}, \mathbf{B}\}$ is the moving orthonormal unit triad following the thalweg. $\tilde{\zeta}$ is the slope angle of the thalweg with the horizontal. The depth of the avalanche in this section is represented by a height function $h(x, y, t)$ and is measured in the radial direction. Right: Debris flow passing through the transition into the run-out zone in a vertical plane containing the thalweg of the valley. In this picture, $x_{l}$ and $x_{r}$ are the left and right end points of the continuous transition between the straight inclined upper part with inclination angle $\tilde{\zeta}_{0}$ and the horizontal run-out in the valley.
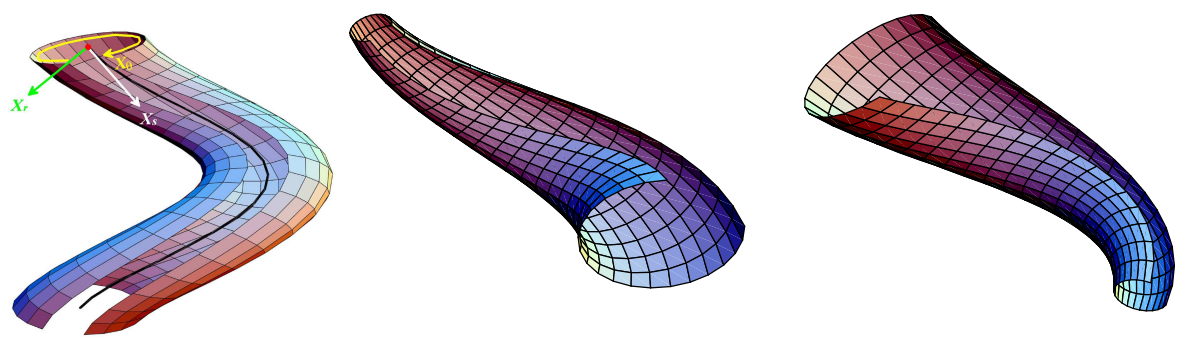

Fig. 2. Left: A representation of a curved and twisted channel, a reference curve and the tangent vectors along the coordinate lines. The dark line along the channel is the axis of the channel; following the notation of Fig. $1 X_{s} \sim \mathbf{T}(x), X_{r} \sim \mathbf{N}(x), X_{\theta} \sim \mathbf{B}(x)$. Middle: Diverging, and Right: converging curved and twisted channels that can be used in the transportation of the granular and debris flow materials.

paper explicitly include curvature and torsion effects in a systematic manner. This makes the extended model amenable to realistic debris motions down arbitrary guiding topographies. This can be accomplished by coordinate transformation. Different from and extending the original SH-theory and all their previous extensions (e.g., Gray et al., 1999; Pudasaini et al., 2003) a moderately curved and twisted space curve is used to define an orthogonal curvilinear coordinate system. The governing balance laws of mass and momentum are written in these coordinates.

\section{Governing equations for single-phase dry granular avalanche}

If the interstitial fluid stress $\mathbf{T}_{f}$ in (7) is zero then Eqs. (7) and (8) together with the solid stress Eq. (9) and the boundary conditions of Sect. 2.3 describe the field equations for single phase avalanching motion. In this section we present the model equations for a single-phase dry granular avalanche.

Pudasaini and Hutter (2003) formulated the balance laws of mass and momentum as well as the boundary conditions in slope-fitted curvilinear coordinates of mountain surfaces, non-dimensionalised the equations and averaged them over the depth of the avalanche. The final balance laws of mass, and momentum in the down-slope and cross-slope directions take the forms (correct to $O\left(\varepsilon^{1+\gamma}\right), 0<\gamma<1$ )

$$
\begin{aligned}
& \frac{\partial h}{\partial t}+\frac{\partial}{\partial x}(h u)+\frac{\partial}{\partial y}(h v)=0, \\
& \frac{\partial}{\partial t}(h u)+\frac{\partial}{\partial x}\left(h u^{2}\right)+\frac{\partial}{\partial y}(h u v)=h s_{x}-\frac{\partial}{\partial x}\left(\frac{\beta_{x} h^{2}}{2}\right), \\
& \frac{\partial}{\partial t}(h v)+\frac{\partial}{\partial x}(h u v)+\frac{\partial}{\partial y}\left(h v^{2}\right)=h s_{y}-\frac{\partial}{\partial y}\left(\frac{\beta_{y} h^{2}}{2}\right),
\end{aligned}
$$

where $h$ is the depth of the avalanche measured normal to the reference surface and the factors $\beta_{x}$ and $\beta_{y}$ are defined as

$\beta_{x}=-\varepsilon g_{z} K_{x}, \quad \beta_{y}=-\varepsilon g_{z} K_{y}$.

The terms $s_{x}$ and $s_{y}$ represent, respectively, the net driving accelerations in the down-slope and cross-slope directions and are given by

$$
\begin{aligned}
& s_{x}=g_{x}-\frac{u}{|\mathbf{u}|} \tan \delta\left(-g_{z}+\lambda \kappa \eta u^{2}\right)+\varepsilon g_{z} \frac{\partial b}{\partial x}, \\
& s_{y}=g_{y}-\frac{v}{|\mathbf{u}|} \tan \delta\left(-g_{z}+\lambda \kappa \eta u^{2}\right)+\varepsilon g_{z} \frac{\partial b}{\partial y},
\end{aligned}
$$


in which $|\mathbf{u}|=\sqrt{u^{2}+v^{2}}$ is the magnitude of the velocity field tangential to the reference (basal) topography and $\left(-g_{z}+\lambda \kappa \eta u^{2}\right)$ is the normal stress at the bed. $\lambda \kappa$ is the local curvature of the thalweg, whilst

$\eta=\cos \left(\theta+\varphi(x)+\varphi_{0}\right)$,

where $\varphi(x)=-\int_{x_{0}}^{x} \tau\left(x^{\prime}\right) d x^{\prime}$ gives the accumulation of the torsion of the thalweg from an initial position $x_{0}$ and $\varphi_{0}$ is a constant. $g_{x}, g_{y}$ and $g_{z}$ are the projected components of the gravitational acceleration along the down-slope, crossslope and normal directions of the channel, for explicit computation see, Pudasaini and Hutter (2003). The aspect ratio $\varepsilon$, and the measure of curvature relative to the typical debris flow length, $\lambda$, are both small numbers given by the scales $[L],[H],[R]: \varepsilon=[H] /[L], \lambda=[L] /[R]$, that are used to non-dimensionalise the equations. Here, $[L]$ is the typical avalanche length, $[H]$ is the typical avalanche height and $[R]$ is a typical radius of curvature of the channel. The basal topography (which is the elevation of the real topography from the reference surface $z=0$, and includes the small-scale geometric features of the bed topography) will be denoted by $z=b(x, y)$.

The first terms on the right-hand side of (19) and (20) are the gravitational accelerations in the down- and cross-slope directions, respectively. The second terms represent the dry Coulomb friction in which the normal tractions comprise of the overburden pressure $\left(-g_{z}\right)$ plus a contribution due to the curvature and torsion of the master curve $\left(\lambda \kappa \eta u^{2}\right)$. Finally, the third terms are the projections of the topographic variations along the normal direction. $K_{x}$ and $K_{y}$ in (18) are called the earth pressure coefficients. Elementary geometrical arguments and Mohr's circles may be used to determine these values as functions of the internal $(\phi)$ and basal $(\delta)$ angles of friction, (Hutter et al., 1993), viz.,

$K_{x}=K_{x_{\mathrm{act} / \mathrm{pass}}}=2 \sec ^{2} \phi\left(1 \mp \sqrt{1-\cos ^{2} \phi \sec ^{2} \delta}\right)-1$,

$(\partial u / \partial x) \gtrless 0$,

$K_{y}=K_{y_{\text {act } / \mathrm{pass}}}=\frac{1}{2}\left(K_{x}+1 \mp \sqrt{\left(K_{x}-1\right)^{2}+4 \tan ^{2} \delta}\right)$,

$(\partial v / \partial y) \gtrless 0$,

where $K_{x}$ and $K_{y}$ are active during dilatational motion (upper sign) and passive during compressional motion (lower sign). We note that ignoring the $O(\varepsilon)$-contributions in (15)(20) reduces the equations to a mass point model and does not allow determination of the deformation of the pile. The dynamics of these equations will also be discussed in Sect. 6 in the context of debris flows. To describe the debris flow, this model must formally be altered only by adding the pore fluid stress.

\section{Evolution and inclusion of the pore fluid stress}

The evolution of the pore fluid stress is crucial in modelling debris flow phenomena. Here we will not present the entire calculation but only write the most important steps for the inclusion of the fluid stress into the model equations of Sect. 4. The routine procedure for the coordinate transformation, non-dimensionalisation, depth-integration, constitutive relations (for the solid phase) and assumption about the nearly uniform velocity profile through the debris flow depth can be found in Pudasaini and Hutter (2003). We will follow the spirit of their paper.

\subsection{Contributions due to fluid stress}

Using the orthogonal coordinates displayed in Figs. 1 and 2 , and with a scaling and dimensional analysis as in Pudasaini and Hutter (2003) the new contributions due to the fluid stress (10) which we must add in the down-slope, cross-slope and normal components of the momentum balance equations of the single-phase dry granular material (see, Eqs. (4.5)(4.7) in Pudasaini and Hutter, 2003) are, respectively

$$
\varepsilon\left[\frac{\partial p}{\partial x}-\frac{2}{N_{R}} \frac{\partial^{2} \tilde{u}}{\partial x^{2}}-\frac{1}{N_{R}} \frac{\partial}{\partial y}\left(\frac{\partial \tilde{v}}{\partial x}+\frac{\partial \tilde{u}}{\partial y}\right)-\frac{1}{\varepsilon^{2} N_{R}} \frac{\partial^{2} \tilde{u}}{\partial z^{2}}\right]+O\left(\varepsilon^{1+\gamma}\right),
$$

$$
\varepsilon\left[\frac{\partial p}{\partial y}-\frac{2}{N_{R}} \frac{\partial^{2} \tilde{v}}{\partial y^{2}}-\frac{1}{N_{R}} \frac{\partial}{\partial x}\left(\frac{\partial \tilde{v}}{\partial x}+\frac{\partial \tilde{u}}{\partial y}\right)-\frac{1}{\varepsilon^{2} N_{R}} \frac{\partial^{2} \tilde{v}}{\partial z^{2}}\right]+O\left(\varepsilon^{1+\gamma}\right),
$$

$\frac{\partial p}{\partial z}+O\left(\varepsilon^{1+\gamma}\right)$

These are dimensionless local expressions and $\tilde{u}, \tilde{v}$ are the local dimensionless velocity components along the downslope and cross-slope directions, respectively, $p$ is the dimensionless fluid pressure and $N_{R}$ is the quasi-Reynolds number which is the fluid volume fraction weighted Reynolds number (as introduced by Iverson and Denlinger, 2001) defined as

$N_{R}=\frac{\sqrt{g L} \rho H}{v_{f} \mu_{f}}$,

where $g$ is the gravity acceleration, $L$ and $H$ are scales used in the non-dimensionalisation, the typical extent and height of the debris flow, $v_{f}$ is the volume fraction and $\mu_{f}$ the viscosity of the fluid. A typical value of $N_{R}$ is on the order of $10^{5}-10^{6}$. In the derivation of $(23)-(25)$, some simplifications have been made, one being that the volume fraction of the fluid, $v_{f}$ is independent of $z$. For complete list of these, see Pudasaini and Hutter (2003).

\subsection{Fluid pressure at the bed}

Due to the shallowness assumption the momentum equation perpendicular to the reference surface is reduced to a balance between the normal derivative of the total normal stress in the normal direction and the mixture (debris) weight in this direction. Adding the fluid contribution (25) to the normal component of the local momentum equation for single-phase 
granular flow (Eq. (4.7), Pudasaini and Hutter, 2003) we obtain the following equation for the pressure distribution in the mixture due to the solid and the fluid

$\frac{\partial p}{\partial z}+\frac{\partial}{\partial z}\left(T_{s(z z)}\right)=g_{z}-\lambda \kappa \eta \tilde{u}^{2}+O(\varepsilon)$.

Integrating this from the surface $z=s(x, y, t)$ to the depth $z$, thereby setting $\tilde{u} \simeq u+O(\varepsilon)$, yields

$p+T_{s(z z)}=\left(-g_{z}+\lambda \kappa \eta u^{2}\right)(s-z)+O(\varepsilon)$.

Therefore, the total pressure at the bed, $z=b(x, y, t)$, is

$p^{b}+T_{s(z z)}^{b}=\left(-g_{z}+\lambda \kappa \eta u^{2}\right) h+O(\varepsilon)$

where $h(x, y, t)=s(x, y, t)-b(x, y, t)$ is the depth of the debris flow.

The fluid pressure is assumed to vary linearly through depth which is also consistent with (28) in the normal direction. The total stress on the bed is now decomposed into two parts, the fluid and solid pressures, as follows:

$$
\begin{aligned}
& p^{b}=\Lambda_{f}\left(-g_{z}+\lambda \kappa \eta u^{2}\right) h+O(\varepsilon), \\
& T_{s(z z)}^{b}=\left(1-\Lambda_{f}\right)\left(-g_{z}+\lambda \kappa \eta u^{2}\right) h+O(\varepsilon),
\end{aligned}
$$

corresponding to the fluid and solid phase pressures (see, e.g., Iverson and Denlinger, 2001; Hubbert and Rubey, 1959). Here, $\Lambda_{f} \in(0,1)$ is a continuous parameter that may depend on several factors such as the debris flow height, time and diffusion of the basal pore pressure along the mixture body from its front to tail. All these are functions of $x$, $y$ and $t$ but not $z$. So, $\Lambda_{f} \neq \Lambda_{f}(\cdot, z)$. Moreover, the limit $\Lambda_{f}=1$ implies zero basal effective stress or complete liquefaction (around the rear end of the debris body) and $\Lambda_{f}=0$ for dry granular flow (e.g. in the vicinity of the front of the debris flow surge). This means that this parameter is mathematically analogous to the fluid volume fraction $v_{f}$. In thermodynamic nonclassical solid-fluid-mixture theories separations like (30) and (31) are referred to as impositions of pressure equilibrium. They are one possible imposition, but other possibilities such as $\Lambda_{f}^{p}$, $\left(1-\Lambda_{f}^{p}\right), p>0$ are equally justified. The separation (30), (31) of the total stress is of constitutive nature and bears the advantages and disadvantages which all such equations exhibit. It is also evident from (30) that $\Lambda_{f}$ is the ratio between the pore fluid pressure at the bed and the total normal pressure of the debris mass in the normal direction. Measurements at the base of experimental flows show that coarse-grained surge fronts have little or no pore fluid pressure. In contrast, finer-grained thoroughly saturated debris behind surge fronts is nearly liquefied by high pore pressure (see Iverson, 1997; Iverson and Denlinger, 2001). So, parameterisation of $\Lambda_{f}$ is very crucial in the description of the dynamics of debris flow surges. Needless to say that here as everywhere else grain size separation is neglected.
5.3 Modification of the friction law and earth pressure coefficients

We must modify the Coulomb friction law and the earth pressure coefficient of the Mohr-Coulomb yield criterion according to the effect of the pore fluid pressure distribution. We identify the fluid normal stress as the pore fluid pressure. As before, we assume that the pore fluid pressure and the solids stress $T_{S(z z)}$ both vary linearly from their maxima at the base to zero at the free surface of the flow. Equation (31) thus implies that the depth-averaged normal solid stress takes the form

$$
\begin{aligned}
\bar{T}_{s(z z)} & =\frac{1}{2}\left(1-\Lambda_{f}\right)\left(-g_{z}+\lambda \kappa \eta u^{2}\right) h+O(\varepsilon) \\
& =-\frac{1}{2} g_{z}\left(1-\Lambda_{f}\right) h+O\left(\varepsilon^{\gamma}\right) .
\end{aligned}
$$

Note that, as in Pudasaini and Hutter (2003), $\lambda=O\left(\varepsilon^{\gamma}\right)$, $0<\gamma<1$ is assumed to have equations correct to $O\left(\varepsilon^{1+\gamma}\right)$. We further assume that the down-slope and cross-slope solid stresses vary linearly with the normal solid stress through the avalanche depth. This is achieved to leading order by the expressions

$T_{s(x x)}=K_{x} T_{s(z z)}+O\left(\varepsilon^{\gamma}\right)$,
$T_{s(y y)}=K_{y} T_{s(z z)}+O\left(\varepsilon^{\gamma}\right)$.

From (32) it follows that the depth-averaged down-slope and cross-slope solid stresses are given by

$\bar{T}_{s(x x)}=-\frac{1}{2} K_{x} g_{z}\left(1-\Lambda_{f}\right) h+O\left(\varepsilon^{\gamma}\right)$,

$\bar{T}_{s(y y)}=-\frac{1}{2} K_{y} g_{z}\left(1-\Lambda_{f}\right) h+O\left(\varepsilon^{\gamma}\right)$.

This implies that the factors $\beta_{x}$ and $\beta_{y}$ in (18) must be modified by the expression

$\beta_{x}=-\varepsilon g_{z} K_{x}\left(1-\Lambda_{f}\right), \quad \beta_{y}=-\varepsilon g_{z} K_{y}\left(1-\Lambda_{f}\right)$.

Similarly, in (19)-(20) the normal solid stress at the bed $\left(-g_{z}+\lambda \kappa \eta u^{2}\right)$ must be replaced by (31) for the solid-fluid mixture. Furthermore, the detailed basal topographic effects $\varepsilon g_{z} \partial b / \partial x$ and $\varepsilon g_{z} \partial b / \partial y$ in (19)-(20) must be replaced by $\left(1-\Lambda_{f}\right) \varepsilon g_{z} \partial b / \partial x$ and $\left(1-\Lambda_{f}\right) \varepsilon g_{z} \partial b / \partial y$, respectively (see, Pudasaini and Hutter, 2003).

Finally, in order to distribute the topographic effects and gravitational driving forces to the solid and fluid components we decompose the components of gravitational accelerations as follows:

$g_{x}=g_{x(s)}+g_{x(f)}=\left(1-\Lambda_{f}\right) g_{x}+\Lambda_{f} g_{x}$,

$g_{y}=g_{y(s)}+g_{y(f)}=\left(1-\Lambda_{f}\right) g_{y}+\Lambda_{f} g_{y}$.

Note that since we are using depth-integrated equations we do not need to decompose $g_{z}$.

\subsection{Depth integration}

Integrating (23) and (24) by repeatedly applying the Leibniz rule (to change the order of integration and differentiation) through the debris flow depth yields the following stresses 
due to the fluid in the pores of the mixture material along the $x$ and $y$ coordinate directions, respectively

$\varepsilon\left[\left\{\frac{\partial}{\partial x}\left(p^{b} h / 2\right)+p^{b} \frac{\partial b}{\partial x}\right\}-\frac{h}{N_{R}}\left\{2 \frac{\partial^{2} u}{\partial x^{2}}+\frac{\partial^{2} v}{\partial y \partial x}+\frac{\partial^{2} u}{\partial y^{2}}-\frac{3 u}{\varepsilon^{2} h^{2}}\right\}\right]+O\left(\varepsilon^{1+\gamma}\right)$,

$\varepsilon\left[\left\{\frac{\partial}{\partial y}\left(p^{b} h / 2\right)+p^{b} \frac{\partial b}{\partial y}\right\}-\frac{h}{N_{R}}\left\{2 \frac{\partial^{2} v}{\partial y^{2}}+\frac{\partial^{2} u}{\partial x \partial y}+\frac{\partial^{2} v}{\partial x^{2}}-\frac{3 v}{\varepsilon^{2} h^{2}}\right\}\right]+O\left(\varepsilon^{1+\gamma}\right)$

where $p^{b}$ is the fluid pressure at the bed defined by (30) whilst $u, v$ are the depth-averaged velocity components in the $x$ and $y$ directions, respectively.

\section{Model equations for two-phase debris flows}

\subsection{Initial boundary value problem}

Incorporating all new effects emerging from the interstitial fluid and detailed by (23)-(38), the granular avalanche Eqs. (15)-(20) become generalised model equations for twophase mixture debris flows sliding and deforming down arbitrarily curved and twisted channels. The governing equations read

$$
\begin{aligned}
& \frac{\partial h}{\partial t}+\frac{\partial}{\partial x}(h u)+\frac{\partial}{\partial y}(h v)=0, \\
& \frac{\partial}{\partial t}(h u)+\frac{\partial}{\partial x}\left(h u^{2}\right)+\frac{\partial}{\partial y}(h u v)=h s_{x}-\frac{\partial}{\partial x}\left(\frac{\beta_{x} h^{2}}{2}\right), \\
& \frac{\partial}{\partial t}(h v)+\frac{\partial}{\partial x}(h u v)+\frac{\partial}{\partial y}\left(h v^{2}\right)=h s_{y}-\frac{\partial}{\partial y}\left(\frac{\beta_{y} h^{2}}{2}\right),
\end{aligned}
$$

which remain formally the same as those for single-phase dry granular avalanche, (15)-(17), but for two-phase debris flows with the following specifications

$$
\begin{aligned}
& s_{x}=s_{x(s)}+s_{x(f)}, \quad s_{y}=s_{y(s)}+s_{y(f)}, \\
& \beta_{x}=-\varepsilon\left(1-\Lambda_{f}\right) g_{z} K_{x}, \quad \beta_{y}=-\varepsilon\left(1-\Lambda_{f}\right) g_{z} K_{y}, \\
& s_{x(s)}=\left(1-\Lambda_{f}\right)\left\{g_{x}-\frac{u}{|\mathbf{u}|} \tan \delta\left(-g_{z}+\lambda \kappa \eta u^{2}\right)+\varepsilon g_{z} \frac{\partial b}{\partial x}\right\}, \\
& s_{y(s)}=\left(1-\Lambda_{f}\right)\left\{g_{y}-\frac{v}{|\mathbf{u}|} \tan \delta\left(-g_{z}+\lambda \kappa \eta u^{2}\right)+\varepsilon g_{z} \frac{\partial b}{\partial y}\right\}, \\
& s_{x(f)}=\Lambda_{f} g_{x}-\varepsilon\left[\frac{1}{h} \frac{\partial}{\partial x}\left(\frac{p^{b} h}{2}\right)+\frac{p^{b}}{h} \frac{\partial b}{\partial x}-\frac{1}{N_{R}}\left\{2 \frac{\partial^{2} u}{\partial x^{2}}+\frac{\partial^{2} v}{\partial y \partial x}+\frac{\partial^{2} u}{\partial y^{2}}-\frac{3 u}{\varepsilon^{2} h^{2}}\right\}\right],
\end{aligned}
$$

$$
s_{y(f)}=\Lambda_{f} g_{y}-\varepsilon\left[\frac{1}{h} \frac{\partial}{\partial y}\left(\frac{p^{b} h}{2}\right)+\frac{p^{b}}{h} \frac{\partial b}{\partial y}-\frac{1}{N_{R}}\left\{2 \frac{\partial^{2} v}{\partial y^{2}}+\frac{\partial^{2} u}{\partial x \partial y}+\frac{\partial^{2} v}{\partial x^{2}}-\frac{3 v}{\varepsilon^{2} h^{2}}\right\}\right],
$$

in which $K_{x}, K_{y}$ are given by (22). Note that, although the basic solid-fluid mixture mass and momentum balance Eqs. (7) and (8) are not in conservative form, the final model equations presented here are in conservative form with source terms ${ }^{2}$. Dimensional analysis, depth integration and ordering processes made it possible to transform these equations from non-conservative to conservative form.

Given the material parameters $\delta$ and $\phi$ and the elevation of the basal topography, $b=b(x, y)$, above the curved reference surface, the viscosity and the volume fraction of the pore fluid, mixture density and fluid pressure distribution at the bed, Eqs. (39)-(47) allow $h, u$ and $v$ to be computed as functions of space and time, once appropriate initial and boundary conditions are prescribed. $h$ is the debris flow depth, and $(u, v)$ are the depth-averaged velocity components parallel to the flow surface. As initial condition one commonly prescribes the geometry and velocity distributions of the debris flow at the initial time, usually for a mass at rest.

6.2 The physics of debris flow described by the present model equations

The model equations presented in this section represent various physical properties of debris flows. We outline some of them as follows:

(i) By setting $\Lambda_{f}=0$ and $N_{R} \rightarrow \infty$ (i.e., ignoring the viscous terms), these model equations reduce to the extended avalanche model equations of Pudasaini and Hutter (2003). On the other hand, when setting $\Lambda_{f}=1$ (i.e., complete liquefaction at the bed and thus zero basal effective stress of the solid) these equations reduce to Boussinesq-type hydraulic (shallow-water) equations with a purely viscous dissipation, e.g., appropriate for a slurry. For intermediate cases the equations indicate a combination of Coulomb friction and viscous dissipation that changes in response to the spatial and temporal changes of the pore pressure.

(ii) Due to the viscous fluid stress effects the equations contain viscous terms via $s_{x(f)}$ and $s_{y(f)}$. Therefore, they are no longer hyperbolic, but hyperbolic-parabolic; they degenerate into hyperbolic equations for single-phase avalanche flows of dry granular materials when the effect of the pore fluid is ignored.

(iii) The model equations involve three non-dimensional parameters, viz., $\varepsilon, \lambda$ and $N_{R}$. (a) The first two are purely geometric parameters and indicate no scale dependence. (b) In contrast, the third parameter, $N_{R}=\rho H \sqrt{g L} / v_{f} \mu_{f}$, serves as a dynamical scaling factor that is analogous to the Reynolds number in Newtonian fluid. Therefore, the debris flow equations are

\footnotetext{
${ }^{2}$ Strictly speaking, the expressions within the square brackets in (46)-(47) are physically not source terms, but in order to maintain the structure of the debris flow equations as those for dry granular flows (15)-(20) we arranged these terms into the source terms.
} 
scale-dependent. Moreover, if we adopt an advectiondiffusion equation (see Sect. 9.4.2) as done by Iverson and Denlinger (2001) for the determination of $p^{b}$ (or $\Lambda_{f}$ ) then, a new parameter $D$, the so-called pore pressure diffusivity, also enters into consideration as a new dynamical parameter. In computations, in order not to distort the geometry of the slide, we shall choose $\varepsilon=\lambda=1$ and thus $L=H=R$.

(iv) The order of different terms in (46) and (47) should be understood as follows. First, let us consider (46). Test simulations reveal that in debris flow dynamics the effect of $\left(2 \partial^{2} u / \partial x^{2}+\partial^{2} v / \partial y \partial x+\partial^{2} u / \partial y^{2}\right)$ is negligible as compared to $3 u / \varepsilon^{2} h^{2}$. On the other hand, typically, $N_{R} \varepsilon^{2}$ is of $O(1)$. Therefore, $\frac{1}{N_{R}}\left\{\left(2 \partial^{2} u / \partial x^{2}+\partial^{2} v / \partial y \partial x+\partial^{2} u / \partial y^{2}\right)-3 u / \varepsilon^{2} h^{2}\right\}$ is also of $O(1)$, consistent with the order of the other terms of (46). A similar analysis holds true for (47).

(v) For geophysical debris flows typical values of $\varepsilon$ and $N_{R}$ are $10^{-3}$ and $10^{6}$, respectively. We have already seen in single-phase avalanche equations that order $\varepsilon$ terms must be retained in the final governing equations. This request is even more intensified for debris flow modelling. The lowest approximation (i.e., $O(1)$ ) excludes the effect of the pore pressure, as is evident from (46), (47). Iverson and Denlinger (2001) have also concluded this fact.

(vi) An expression for the evolution of the basal pressure $p^{b}$ can be obtained by an advection-diffusion equation for $p^{b}$ that is responsible for the distribution of the pore pressure along the bed of the debris flow. Iverson and Denlinger (2001) expressed $\Lambda_{f}$ as a function of the mixture height, time and the diffusivity of the mixture. Alternatively, Savage and Iverson (2003), coupled the pore pressure evolution equation with the mass and momentum balance equation to describe debris flow surge dynamics over a one-dimensional rough incline. Here, first, we will take some constant values, and then a bilinear parameterisation of $\Lambda_{f}$ in time and space for simulation. This simple parameterisation can also produce debris flow surges as discussed by Iverson and Denlinger (2001) and Savage and Iverson (2003). In applications, one may couple these or other parameterisations or evolution equations for pore pressure of the fluid (as done in Sect. 9.4.2) with the present model equations to describe debris flow surges down more general mountain terrains.

(vii) The curvature, $\lambda \kappa$, and torsion, $\eta$, effects of the geometry enter in the model equations directly via the Coulomb friction terms in (44)-(45), but they are equally indirectly contained in the viscous terms (46)(47) via the pressure, $p^{b}$, at the bed, see (30). Note that the components of the gravitational accelerations $g_{x}, g_{y}, g_{z}$ also include such effects due to the curvature and torsion of the reference topography (see Pudasaini and Hutter, 2003).

\section{The new features of the model equations}

Equations (39)-(41) constitute a two-dimensional conservative system of equations which entails several advantages over previous model equations of the Savage-Hutter (1989) type for dry granular materials and Iverson (1997) type for mixtures. They are as follows:

(1) The equations systematically include the curvature and torsion of the channelised basal topography. They are written in a slope-fitted general orthogonal curvilinear coordinate system. Therefore, they can be utilised to describe debris flows along non-uniformly curved and twisted channels of general type.

(2) There is a non-zero gravity term $g_{y}$ in the cross-slope direction, see (45) and (47), which takes into account the global effect of topographic variation in the lateral direction. Thus, the lateral motion is explicitly gravity driven, not only indirectly via lateral confinement gradient, i.e., $\partial b / \partial y$. This might be crucial in designing defence structures and when debris flows hit obstructions or are deflected on their ways. The torsion effect, $\eta$ of the topography is included in the net driving force components $s_{x}$ and $s_{y}$. The components of the gravitational acceleration also depend on both the curvature and the torsion of the basal topography, (see Pudasaini and Hutter, 2003). The $y$ coordinate, which was just a straight line in the previous models, is now curved in the cross-slope direction and for a torsion-free master curve (i.e., $\eta=1$ ), which lies in a vertical plane, these model equations exactly degenerate to previous extensions (e.g., Gray et al., 1999).

(3) Similarly, these equations can exactly be reduced to the avalanche equations of Pudasaini and Hutter (2003) as special cases for which the parameters $\Lambda_{f}=0, N_{R} \rightarrow \infty$.

(4) We can form a three-dimensionally curved and twisted channel using down-slope and cross-slope coordinates $x$ and $y$ alone. In principle, it is thus possible to model a given channel by considering its thalweg and by choosing $\theta$ appropriately as a function of the down- and crossslope coordinates. These are new flexibilities of the model equations which are crucial to describe the motion of avalanching debris flows in curved and twisted channels and mountain terrains in a more realistic manner.

(5) The equations of this paper are well structured and put in a standard mathematical form. They enjoy two advantages: First, it is easy to write a numerical code or to extend an existing numerical code available for avalanche flows by including the new additional effects of the viscous pore fluid. Next, from these model equations one can easily distinguish the friction contribution of the solid grains and the viscous effect of the pore fluid and their coupling. This coupling is represented by 
the parameter $\Lambda_{f}$. Similarly, the structure of the equations shows that the granular avalanche equations are of hyperbolic type whereas, due to the pore fluid diffusion, debris flow is governed by hyperbolic-parabolic type partial differential equations.

(6) In the present model the pore pressure at the bed, $p^{b}$, includes effects of curvature and torsion of the bed topography, see (30). Numerical results show that such an effects are substantial, for detailed discussion, see Sect. 9.1 and Fig. 4.

(7) The coordinates of these model equations follow the basal topography. So, a mathematically correct asymptotic analysis could rigorously be performed.

In this sense, one may infer that the above equations should be physically, geometrically and mathematically more suitable than previously proposed models (e.g., Iverson, 1997; Iverson and Denlinger, 2001) for the description of debris flows down arbitrarily curved and twisted channels with variable cross-sections.

\section{Numerical integration techniques}

The debris flow equations (39)-(41) comprise a nonlinear conservation system. Shock formation, possibly diffusively smoothed out, is an essential mechanism in debris flows on an inclined surface merging into a horizontal run-out zone or encountering an obstacle when the velocity becomes subcritical from its supercritical state. Note that the formation of shocks in debris flow depends strongly on the volume fraction of the solid. Therefore, for high sediment concentrations debris flow surge fronts are most likely associated with a jump in the height and velocity field although the rear part of the debris is highly liquefied. This will be discussed in more detail in Sects. 9.3 and 9.4. To produce more accurate and physically reliable solutions of strongly convective nonlinear conservation equations, it is therefore natural to apply conservative high-resolution numerical techniques that are able to resolve the steep gradients of the unknown variables and moving fronts often observed in experiments and field events of avalanches and debris flows. The NOC (NonOscillatory Central) scheme proposed first by Nessyahu and Tadmor (1990) and extended to higher dimensions by Jiang and Tadmor (1998) is implemented to solve the model equations. This is a high resolution shock capturing scheme. The necessary background and full details of this method can be found in the literature (see, e.g., Harten, 1983; Harten et al., 1986; Yee, 1987; Nessyahu and Tadmor, 1990; LeVeque, 1990; Kröner, 1997; Jiang and Tadmor, 1998; Toro, 2001) and its application to avalanches is, e.g., given by Tai et al. (2002), Pudasaini (2003), Pudasaini and Hutter (2003a), Wang et al. (2004) and Pudasaini et al. (2005a, b). We do not further elaborate here on the TVD (Total Variation Diminishing) techniques and optimal choices of limiters and cell reconstructions. Wang et al. (2004) made a careful study investigating its optimal use in avalanche studies of the extended
Savage-Hutter equations. For alternative numerical schemes also see Denlinger and Iverson (2001, 2004), Koschdon and Schäfer (2003), Iverson et al. (2004), Patra et al. (2005) and Vollmöller (2004).

This scheme requires the system to be written in terms of conservative variables, which are the debris flow thickness, $h$ and the depth integrated down- and cross-slope momenta, $m_{x}=h u$ and $m_{y}=h v$. With the vector of conservative variables, $\mathbf{w}=\left(h, m_{x}, m_{y}\right)^{T}$, the model Eqs. (39)-(41) can be written as

$\frac{\partial \mathbf{w}}{\partial t}+\frac{\partial \mathbf{f}(\mathbf{w})}{\partial x}+\frac{\partial \mathbf{g}(\mathbf{w})}{\partial y}=\mathbf{s}(\mathbf{w})$.

The down-slope and cross-slope momentum flux vectors $\mathbf{f}$ and $\mathbf{g}$, and the vector of the source terms $\mathbf{s}$ are given by

$$
\begin{aligned}
& \mathbf{f}=\left(\begin{array}{c}
m_{x} \\
m_{x}^{2} / h+\beta_{x} h^{2} / 2 \\
m_{x} m_{y} / h
\end{array}\right), \\
& \mathbf{g}=\left(\begin{array}{c}
m_{y} \\
m_{x} m_{y} / h \\
m_{y}^{2} / h+\beta_{y} h^{2} / 2
\end{array}\right), \\
& \mathbf{s}=\left(\begin{array}{c}
0 \\
h s_{x} \\
h s_{y}
\end{array}\right) .
\end{aligned}
$$

The terms $\beta_{x}$ and $\beta_{y}$, defined in (43) incorporate the extending and contracting states of the avalanching debris mass through the active and passive earth pressures. Similarly, the source terms $s_{x}$ and $s_{y}$, described in (42), (44)-(47) are of crucial importance as they include the total driving force generated by gravity, friction, curvature, torsion and local details of the basal topography through its gradient terms and the pore pressure distribution of the viscous fluid. They jointly determine the dynamics of the flow.

\section{Debris flow down curved and twisted channels}

The model equations (39)-(41) predict the flow of a debris material over a non-uniformly curved and twisted channel in which the cross-slope curvature (or the channel width) may equally be varying. We will focus on the numerical simulations of such flows, their physical explanation and the analysis and interpretation of the results. The main target is the analysis of the joint effects of curvature, torsion, cross-slope topographic variation and "centrifugal" force and the effects of the pore fluid pressure in the dynamics of a debris flow body down more general channels and topographies. In the examples below, the form of the employed master curve and the variations of the cross sections are still somewhat idealistic. Nevertheless, the examples will disclose features and effects that are quantitatively well understood but could, so far not be explicitly quantised. What we mean here are effects of combinations of pore pressure and geometric effects due to, e.g., curvature and torsion of the thalweg. The results will allow us to judge about the applicability of the 

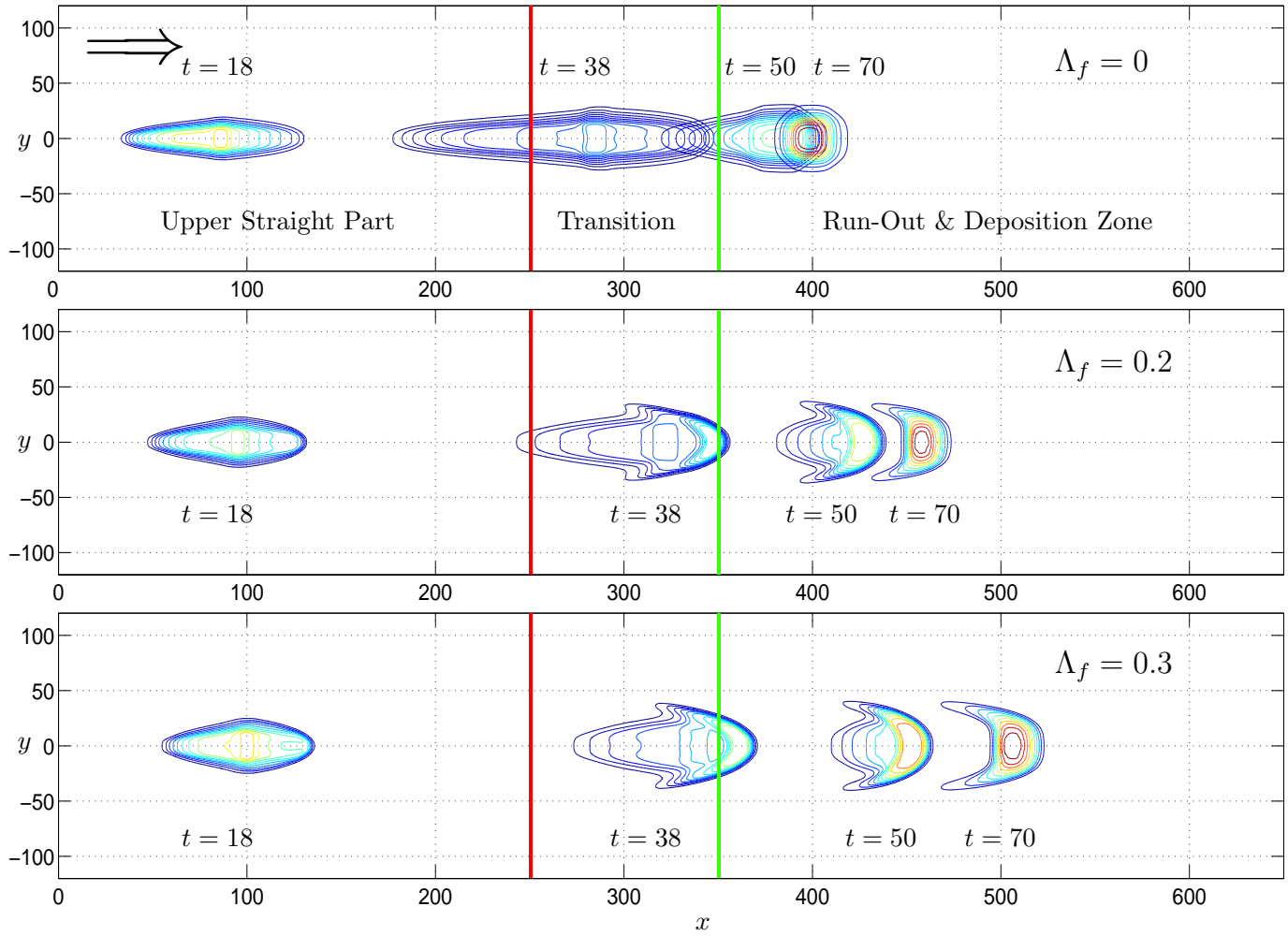

Fig. 3. Contour plots of an avalanching debris mass flowing down a curved chute that is flat in the lateral direction: granular-fluid mixture with fluid component: $\Lambda_{f}=0$ (top), $\Lambda_{f}=0.2$ (middle) and $\Lambda_{f}=0.3$ (bottom). The upper part of the chute $(x<250)$ is inclined at an angle of $45^{\circ}$, the middle part is a transition zone $(250<x<350)$ and the final part $(x>350)$ is horizontal and flat. Time slices: $t=18,38,50,70$. The mass is initially kept in a hemi-spherical cap of radius 6.5 centred at $(23,0)$ and initial velocity is zero. Internal and bed friction angles of the grains are $33^{\circ}$ and $27^{\circ}$, respectively and $N_{R}=3 \times 10^{5}$. All quantities are dimensionless.

new-model equations. On the other hand, they will open a wide spectrum of possibilities for practitioners involved in hazard mapping, risk management and public safety. For single-constituent dry granular avalanches, corresponding results were obtained by Pudasaini et al. (2005a). This paper aims to extend those simulations for two-phase debris flows. In the sequel, we will separately present simulations (i) for a simply curved chute or a flume, and (ii) for continuously curved and twisted channels. We will consider different (variable) volume fractions of the fluid component in order to study the explicit influence of the interstitial fluid in debris flow dynamics. Another important aspect of this paper is the coupling of the advection-diffusion equation, proposed by Iverson and Denlinger (2001) for the determination of the bed fluid pressure, with the model Eqs. (39)-(41), and comparison of model simulations with the laboratory and field experiments.

\subsection{Debris flows down a curved chute}

\section{Geometry of the chute and parameters:}

Let us consider a chute with a flat upper part $(x<250)$ inclined at an angle of $45^{\circ}$, continuous transition zone $(250<x<350)$ and a horizontal, flat, final part $(x>350)$.
Only one curvature in the downhill direction is involved in this configuration. Hence, the chute is laterally flat and torsion-free, i.e. $\eta=1$.

Figure 3 depicts several contour plots of debris flows down such a curved chute in which the ratio between the pore fluid pressure at the bed and the total normal pressure of the debris mass perpendicular to it correspond to $\Lambda_{f}=0$ (top), $\Lambda_{f}=0.2$ (middle) and $\Lambda_{f}=0.3$ (bottom). For all values of $\Lambda_{f}$ (roughly speaking, the volume fraction of the fluid) the contours are plotted for four dimensionless time slices: $t=18,38,50,70$. The mass is initially kept in a hemispherical cap of radius 6.5 centred at $(23,0)$ and the initial velocity is zero. The internal and bed friction angles of the grains are $33^{\circ}$ and $27^{\circ}$, respectively, and the quasi-Reynolds number is taken to be $N_{R}=3 \times 10^{5}$, see Iverson and Denlinger (2001). These parameters will also be used in the remainder of the paper whenever applicable.

\section{Discussion of results:}

Geometric effects: For a simply curved chute, as used here, it is easier to understand the geometric effect on the dynamics of the debris flow. After the release of the mass from its initial rest state the debris flow is accelerating primarily in the longitudinal direction and only a little spread takes place 


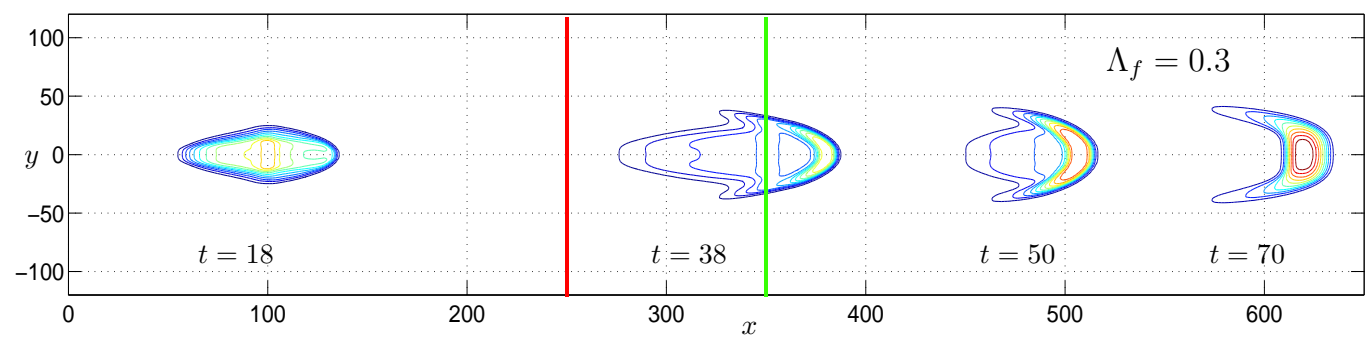

Fig. 4. As in the last panel of Fig. 3 but neglecting the influence of the curvature in the pressure at the bed; manifesting substantial contribution of the term including the curvature in the dynamics of the debris flow.

in the transversal direction, see forms of the masses at time $t=18$ in all three panels in Fig. 3. This is clearly because the flow is driven by gravity. As soon as the mass enters the transition zone due to the longitudinal curvature of the chute, due to the increased friction the body starts extending also in the cross-slope direction. These effects are seen in all panels at time $t=38$. In the run-out zone the height of the pile is always increasing (from $t=50$ to $t=70$ ) and the body comes to a rest, as plotted for time $t=70$. At all times the sliding and deforming debris body is symmetric about the central line $(y=0)$ of the chute. This is evident because the chute is torsion-free.

Effects of the fluid: Our next aim is to study the influence of the fluid component in the dynamics of the debris flow. From the mechanism of the mixture of solid and fluid constituents it is clear that as the value of the parameter $\Lambda_{f}$ increases the contribution of the fluid increases. This is simply due to the fact that the Coulomb friction between the grains is decreased, and that the debris mass is more liquefied. Consequently, with increasing values of the parameter $\Lambda_{f}$ the travel distance of the flow increases dramatically. The fluid presence also implies changes to the form of the body. The forms and positions of the fronts and tails and the curvature of the geometry of the deforming body are explicitly dependent on the value of $\Lambda_{f}$. With increasing value of $\Lambda_{f}$ the increase of the speed of the tail is much faster than the speed of the front (e.g., compare the three panels in Fig. 3 at time $t=38$ ). Further interesting phenomena are in the deposit and in the run-out zone. The top panel shows that the final deposit (time $t=70$ ) for dry granular flow is convex with its center lying before $x=400$, whilst for debris flow, e.g., with $\Lambda_{f}=0.3$, the center of the body lies beyond $x=500$, see the bottom panel; and the form of the deposit has reverse "Barchan dune type" geometry with two "horns" lying on either side of the central line of the chute facing the upstream direction.

From these observations one may draw the inferences that the form and speed of debris flows are explicitly influenced both by the geometry of the topography and the relative volume fractions of the fluid and solid constituents in the mixture.
Effects of the curvature on the pressure at the bed

From Eqs. (30) and (31) one can infer that there is substantial contribution of the curvature and torsion on the pressure at the bed by the term $\lambda \kappa \eta u^{2}$, hence on the flow dynamics. To see this effect quantitatively, we repeat the above simulation with $\Lambda_{f}=0.3$, but without inclusion of the curvature in the expression for the basal normal pressure, i.e., $p^{b}=-\Lambda_{f} g_{z} h$ and $T_{s(z z)}^{b}=-\left(1-\Lambda_{f}\right) g_{z} h$. This shows that the pore fluid pressure at the bed is decreased by the amount $\Lambda_{f}\left(\lambda \kappa \eta u^{2}\right) h$ but at the same time the solid (normal) pressure at the bed is also decreased by $\left(1-\Lambda_{f}\right)\left(\lambda \kappa \eta u^{2}\right) h$. This means that, although the mobility due to the fluid component is decreased (relatively) by $30 \%$, the relative $70 \%$ decrease in the solid normal pressure reduces the Coulomb frictional resistance quite considerably, see (44) and (45), thus substantially increasing the reach of the debris body in the transition zone. Figure 4 displays the effect of the curvature on the pressure at the bed. Parameters chosen for the simulation are as in the last panel of Fig. 3, but the curvature of the bed topography is set to zero. The difference in the dynamics between these two pictures is substantial. Similarly, one can investigate the effect of the torsion on the pressure at the bed.

9.2 Debris flow down non-uniformly curved and twisted channels

At first, we consider helically curved and twisted channels. This is an academic test example, a helix as a master curve so as to form a helically curved and twisted channel with otherwise circular and/or parabolic cross section. Let us consider a circular helix described by

$\mathbf{R}(\vartheta)=(A \cos \vartheta, A \sin \vartheta,-B \vartheta)$,

where $\vartheta$ is the azimuthal angle. The arc length, curvature, torsion and pitch of the helix are given by

$$
\begin{aligned}
& x=\left(A^{2}+B^{2}\right)^{1 / 2} \vartheta, \\
& \kappa=A /\left(A^{2}+B^{2}\right), \\
& \tau=-B /\left(A^{2}+B^{2}\right), \\
& \mathcal{P}=2 \pi B,
\end{aligned}
$$

respectively. 

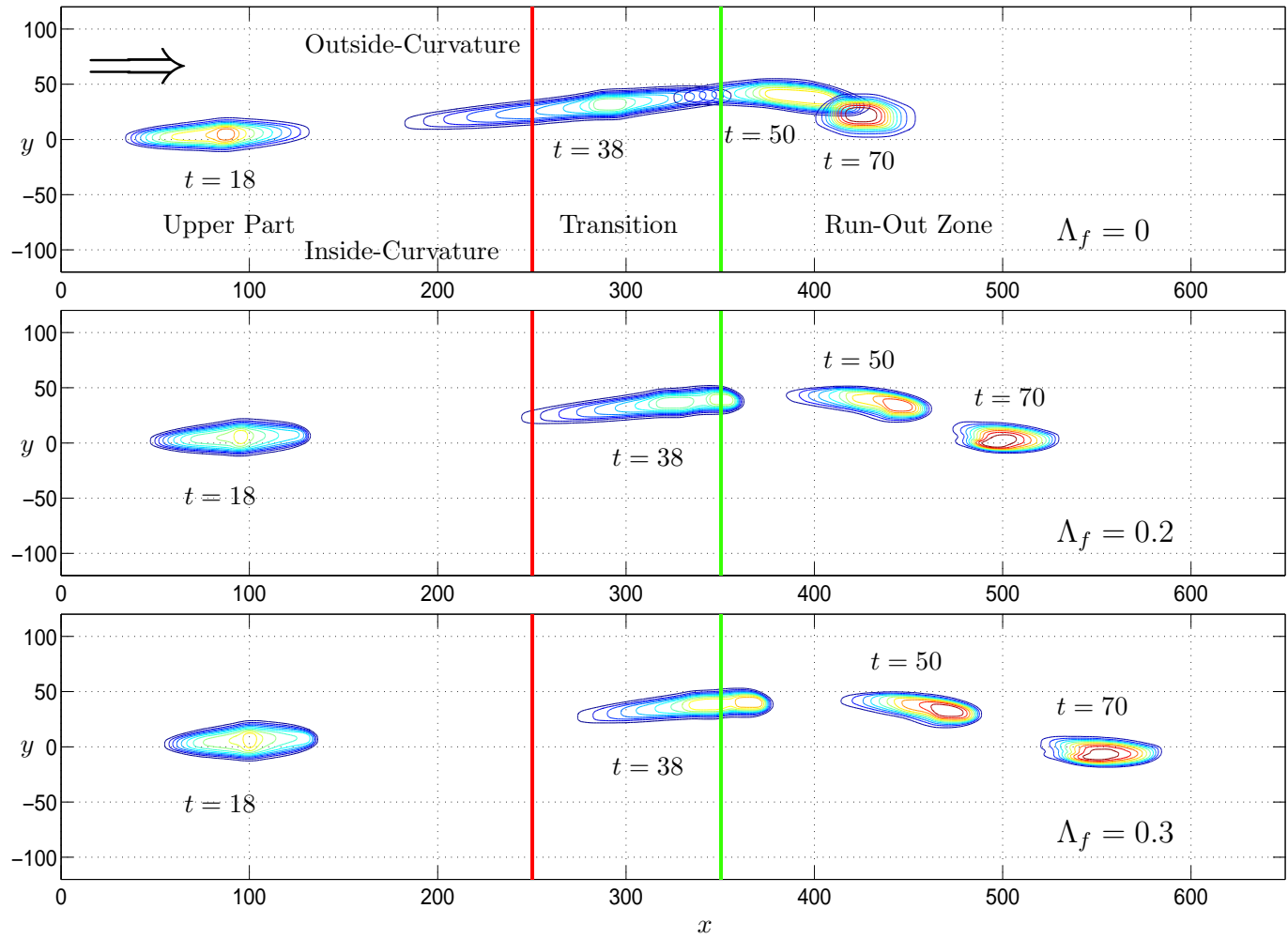

Fig. 5. Contour plots of avalanching debris mass flowing down a curved and twisted channel: granular-fluid mixture with fluid component: $\Lambda_{f}=0$ (top), $\Lambda_{f}=0.2$ (middle) and $\Lambda_{f}=0.3$ (bottom). The upper part of the chute $(x<250)$ is inclined at an angle of $45^{\circ}$, the middle part is a transition zone $(250<x<350)$ and the final part $(x>350)$ is a horizontal channel. Parameter values are: $A_{0}=B=300$, $y \in[-120,120], z_{T}=128$. Time slices: $t=18,38,50,70$. The mass is initially kept in a hemi-spherical cap of radius 6.5 centred at $(23,0)$ and initial velocity is zero. Internal and bed friction angles of the grains are $33^{\circ}$ and $27^{\circ}$, respectively and $N_{R}=3 \times 10^{5}$. All quantities are dimensionless.

Based on the master curve (50) a helically curved and twisted channel is formed. The lateral section of the topography is the intersection of a plane perpendicular to the thalweg of the channel and the channel itself. In the sequel, we will deal with cases in which the transition and run-out zones are included in the geometrical part of the model and that the cross-sectional geometry of the channel is also variable.

In reality channels may be arbitrarily curved and twisted with variable cross-slope curvature and channel width. Realistic flow tracks go from steep to flat regions where the moving masses come to a halt. The geometry must play a crucial role to make the body stand still. The concave curvature of the mountain side increases the bed friction and consequently forces the debris mass to slow down and eventually come to rest. In this subsection we will present debris flow simulations through more general channels which possess run-out zones.

\subsubsection{Variable curvature and torsion}

Consider a channel of which curvature and torsion are redefined with a new expression for $A$ in (51) as follows:

$$
A(x)= \begin{cases}A_{0}, & 0 \leq x \leq x_{l}, \\ A_{0} \exp \left[\left(x-x_{l}\right)^{a}\right], & x_{l} \leq x \leq x_{r}, \\ A_{0} \exp \left[\left(x_{r}-x_{l}\right)^{a}\right], & x \geq x_{r}\end{cases}
$$

where $a$ determines the intensity of the decrease of the curvature and torsion. For the simulations, we have set $a=1$. Equation (52) tells us that the radius of curvature and torsion of the channel increase rapidly as the arc-length $x$ becomes larger than $x_{l}$. Before this transition point, the channel has uniform radius of curvature and torsion. This increase forces the channel quickly to merge (approximately) into a lesser and lesser curved and eventually horizontal channel. This horizontal portion of the channel also forms the run-out zone for the debris. There is a continuous decrease of the curvature and torsion from $x_{l}=250$ to $x_{r}=350$. Then, for $x \geq x_{r}$ the curvature and torsion are always (almost) zero, and thus, the subsequent channel is forming a channelised circular runout. The parameter values are: $A_{0}=300, B=300$, so that the channel is inclined relative to the horizontal at $45^{\circ}$. The radius of curvature in the cross-slope direction is $z_{T}=128$ and $y \in[-120,120]$.

\section{Discussion of results:}

Geometric effects: Figure 5 displays thickness contours of debris flows with three different values of $\Lambda_{f}$, respectively, sliding down through a helically curved and twisted channel with non-uniform curvature and torsion given by (51) and 


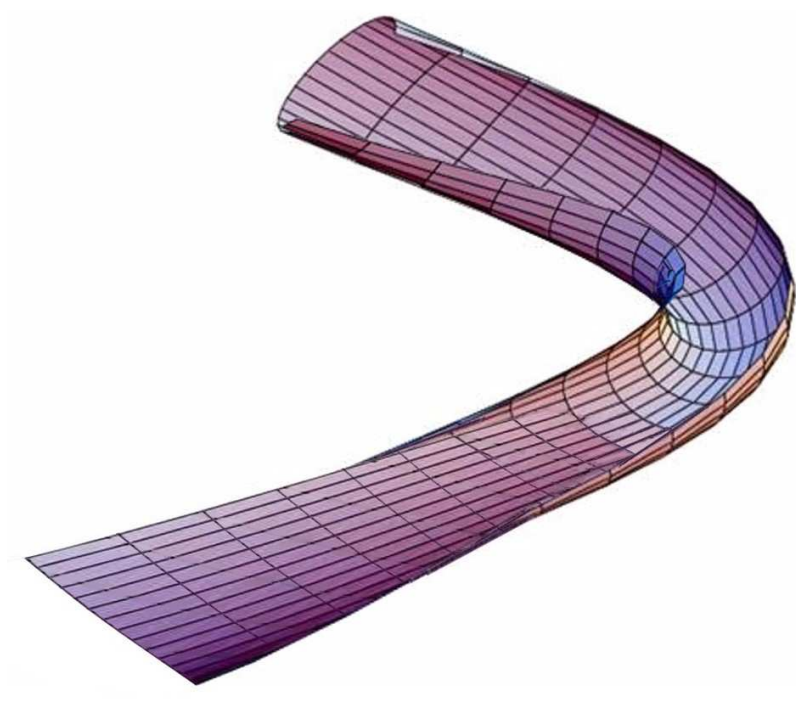

Fig. 6. Curved and twisted channel. The channel has a circular cross-section before the transition and merges continuously into the flat horizontal run-out zone.

(52) and a constant cross-slope channel width ${ }^{3}$. These contours are plotted at the time steps $18,38,50,70$, respectively. As time increases, the debris mass is laterally getting less spread, but, it is rapidly moving outwards from the center line of the channel in the front much more than in the back. This effect can clearly be seen in all three panels at times $t=18$ and $t=38$. This is so because the speed of the front is much larger than that of the tail. Such behaviour of the deforming mass is the joint effect of the curvature, torsion, and the radial acceleration that is modelled in the theory (Eqs. (15)-(17) for dry avalanches, the top panel and Eqs. (39)-(41) for debris flows, the middle and bottom panels) through the gravitational acceleration components $g_{x}, g_{y}, g_{z}$ and the net driving force components $s_{x}, s_{y}$, which include the curvature and torsion of the thalweg, bed topography and the cross-slope curvature of the channel.

Since the curvature and torsion of the channel are continuously decreasing for $x>x_{l}=250$, from $t=38$ onward, the debris mass tends to slow down and turn smoothly towards the central line of the channel due to the confinement gradient in the cross-slope. Corresponding to the decrease of the curvature and torsion, the inclination angle of the chute with the horizontal plane is also continuously decreased. Ultimately, the channel merges into a horizontal circularly curved channel, thus forming a gully-type channelised runout zone. Somewhere in the transition zone the sidewise pressure (due to the lateral component of the gravitational

\footnotetext{
${ }^{3}$ All figures shown for helical chutes are geometrically distorted. The graphs are vertical projections of the chute and debris heaps whose circular-annular geometry is stretched to become straight. Thus, a segment of the annular ring becomes a rectangle of which the top edge is the chute outside and the bottom edge the chute at the inside boundary. This graphical representation is chosen because it is relatively easy to program.
}

force towards the central line) from the channelised bed topography exceeds the force due to the radial acceleration. It leads to a continuous rotation of the body towards the center of the channel. This sidewise pressure is so strong that after $x=350$ the mass comes back to the thalweg of the channel (middle panel) and heads towards the opposite side of the channel (bottom panel). Finally, the body comes to rest at a time prior to $t=70$.

Effect of the fluid: The effect of the fluid is much more pronounced here than in the previous case with debris sliding over a curved but not twisted chute, see Fig. 3. With increasing value of the parameter $\Lambda_{f}$ the debris mass slides faster throughout the channel and travels farther and farther in the run-out zone. Similarly, with the increasing value of $\Lambda_{f}$ the center of mass of the final deposit comes closer and closer to the central line of the channel (compare top and middle panels) and ultimately it crosses the central line (bottom panel). This behaviour of the motion of the body is dominated by both the geometry of the channel and the contribution of the fluid component in the mixture. Since the chute is uniformly channelised from initiation to the run-out zone in the cross-section the mass can not spread in the lateral direction not even in the run-out zone. Instead, it is accumulated and elongated around and along the thalweg of the channel. The channelised topography also does not allow the formation of the Barchan type geometry of the debris mass in the run-out zone.

9.2.2 Decreasing curvature \& torsion, and variable crossslope curvature

Real channels may be diverging or converging (with respect to their channel width or cross-slope curvature) along the downhill direction, see Fig. 2. Therefore, the debris flow theory must be able to deal with more general channels with varying cross-slope curvature. At this point, we simulate the debris flow motion in a channel of which the parameter $A$ is defined by (52) as in the previous case, but, now we vary the channel width starting from its left boundary of the transition zone. This case is more important in geophysical applications because curvature and torsion decrease as one enters into the horizontal run-out zone of a mountain valley. This effect can be achieved by defining a channel which merges continuously into an open flat run-out zone according to

$\theta(x, y)= \begin{cases}y / z_{T}, & 0 \leq x \leq x_{l}, \\ \left(y / z_{T}\right) f(x), & x_{l} \leq x \leq x_{r}, \\ 0^{\circ}, & x \geq x_{r},\end{cases}$

where $z_{T}$ is the distance between the master curve and the thalweg in the upper inclined part of the channel (hence a constant) and $f(x)=\left(1-\left(x-x_{l}\right) /\left(x_{r}-x_{l}\right)\right)^{2}$. Thus, the continuous transition of the parametric function $\theta$ from its higher value $\left(y / z_{T}\right)$ in the upper part to its zero value in the open run-out zone constitutes a three-dimensional channel which has variable curvature both in the longitudinal and lateral directions. Other parameters are as in Sect. 9.2.1. A representative example of such a channel is illustrated in Fig. 6. 

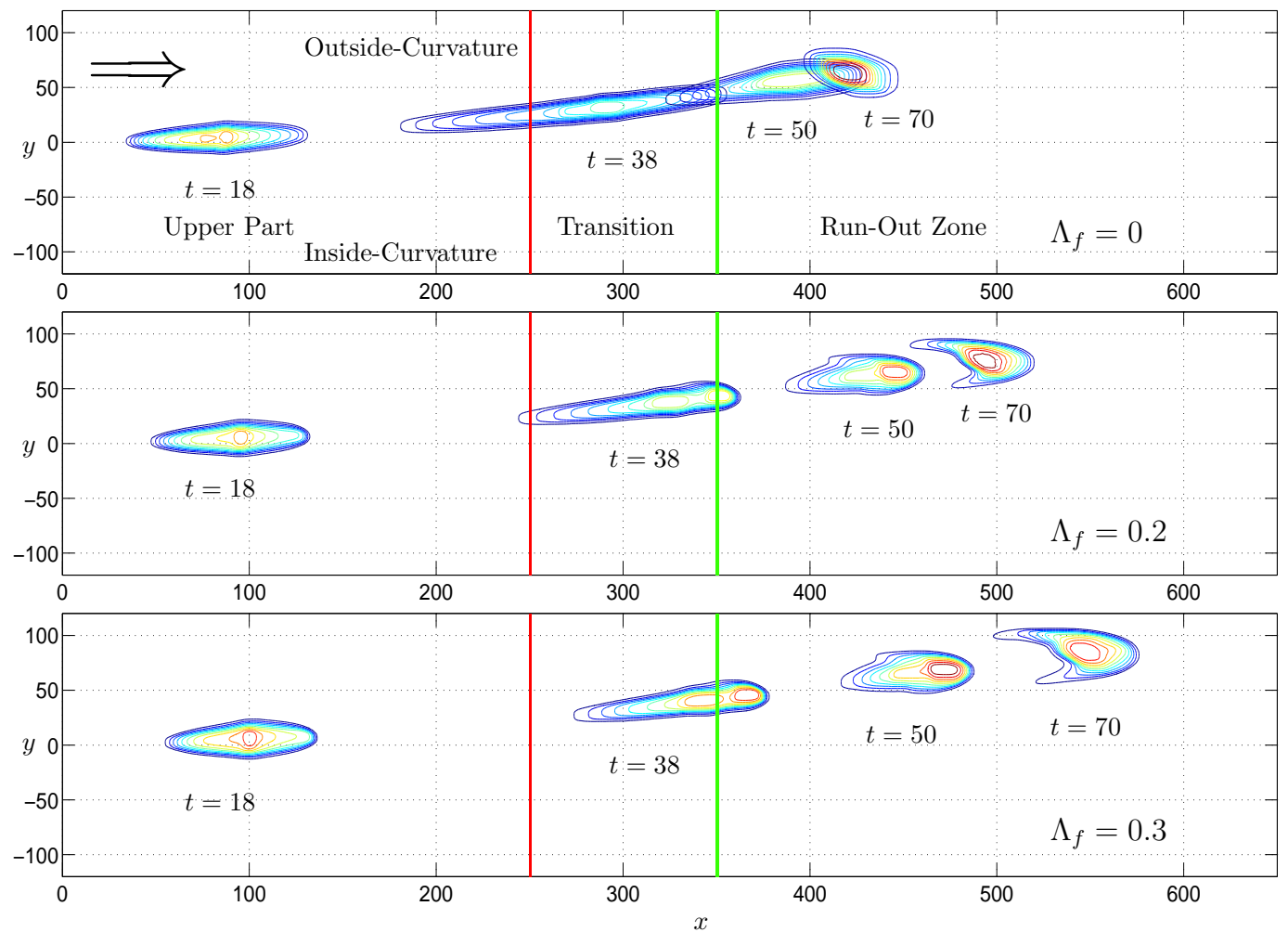

Fig. 7. Contour plots of avalanching debris flowing down a curved and twisted channel: granular-fluid mixture with fluid component: $\Lambda_{f}=0$ (top), $\Lambda_{f}=0.2$ (middle) and $\Lambda_{f}=0.3$ (bottom). The upper part of the chute $(x<250)$ is inclined at an angle of $45^{\circ}$, the middle part is a transition zone $(250<x<350)$ and the final part $(x>350)$ is horizontal and flat. Parameter values are: $B=300$, $A$ is redefined with $A_{0}=300$, the channel width is redefined with variable $\theta, y \in[-120,120], z_{T}=128$. Time slices: $t=18,38,50,70$. The mass is initially kept in a hemi-spherical cap of radius 6.5 centred at $(23,0)$ and initial velocity is zero. Internal and bed friction angles of the grains are $33^{\circ}$ and $27^{\circ}$, respectively and $N_{R}=3 \times 10^{5}$. All quantities are dimensionless.

\section{Discussion of results:}

Geometric effects: Figure 7 depicts the contours of the debris flow motion after its release to the open run-out zone. The graphs describe the deformation of the debris disclosing the subtle reaction of it to the different geometry of the transition and run-out region. Although the inclination of the channel is decreasing after reaching the transition zone, the debris body is heading radially outwards of the flat run-out zone until it comes to rest close to the outside edge of the open channel. The main mechanism for this is that, as soon as the mass enters the runout zone the radial acceleration decreases rapidly, but, since the chute is flattening in the crossslope direction, after the transition zone, the material body moves in the direction of the velocity at the moment directly after the transition, departing away from the central line, and the velocity is decreasing with time due to the bed friction until the debris body comes to rest. The direction and the process of the deposition is in conformity with our physical intuition and expectation.

Effects of the fluid: The most interesting effect of the fluid component can be observed in this figure. Before the transition zone, the dynamics of the flow is exactly the same as in Fig. 5, travel distances in the run-out zones are also sim- ilar. But, the form of the sliding body in the run-out zone is completely different from that for the entirely channelised topography (Fig. 5). Since the channel is gradually opened in the run-out zone the radial acceleration makes it possible to form the Barchan type geometry as in Figs. 3 and 4, but now with the horns pointing obliquely-upstream. Note that with increasing value of the fluid component the aerial coverage of the deposit is also increasing.

\subsection{Variable pore pressure distribution}

A simple parameterisation for pore fluid pressure. The structure of Eqs. (39)-(47) indicates that $\Lambda_{f}$ plays a significant role in the description of the debris flow (see, Iverson and Denlinger, 2001; Savage and Iverson, 2003 for further explanation). Therefore, a proper parameterisation or description of $\Lambda_{f}$ is necessary. In reality, the pore-fluid pressure is not constant but varies with time and along the debris body, usually its value being smaller in the front and larger in the rear part. As a first attempt, to investigate the effect of the variable pore pressure to the dynamics of debris flow we parameterise $\Lambda_{f}$ as

$$
\Lambda_{f}=\Lambda_{f}^{\text {mean }}-\Delta \Lambda_{f} \frac{\left(x-\left(x_{r}+x_{f}\right) / 2\right)}{\left(x_{f}-x_{r}\right)} \frac{\left(t-t_{0}\right)}{\left(t_{\max }-t_{0}\right)},
$$



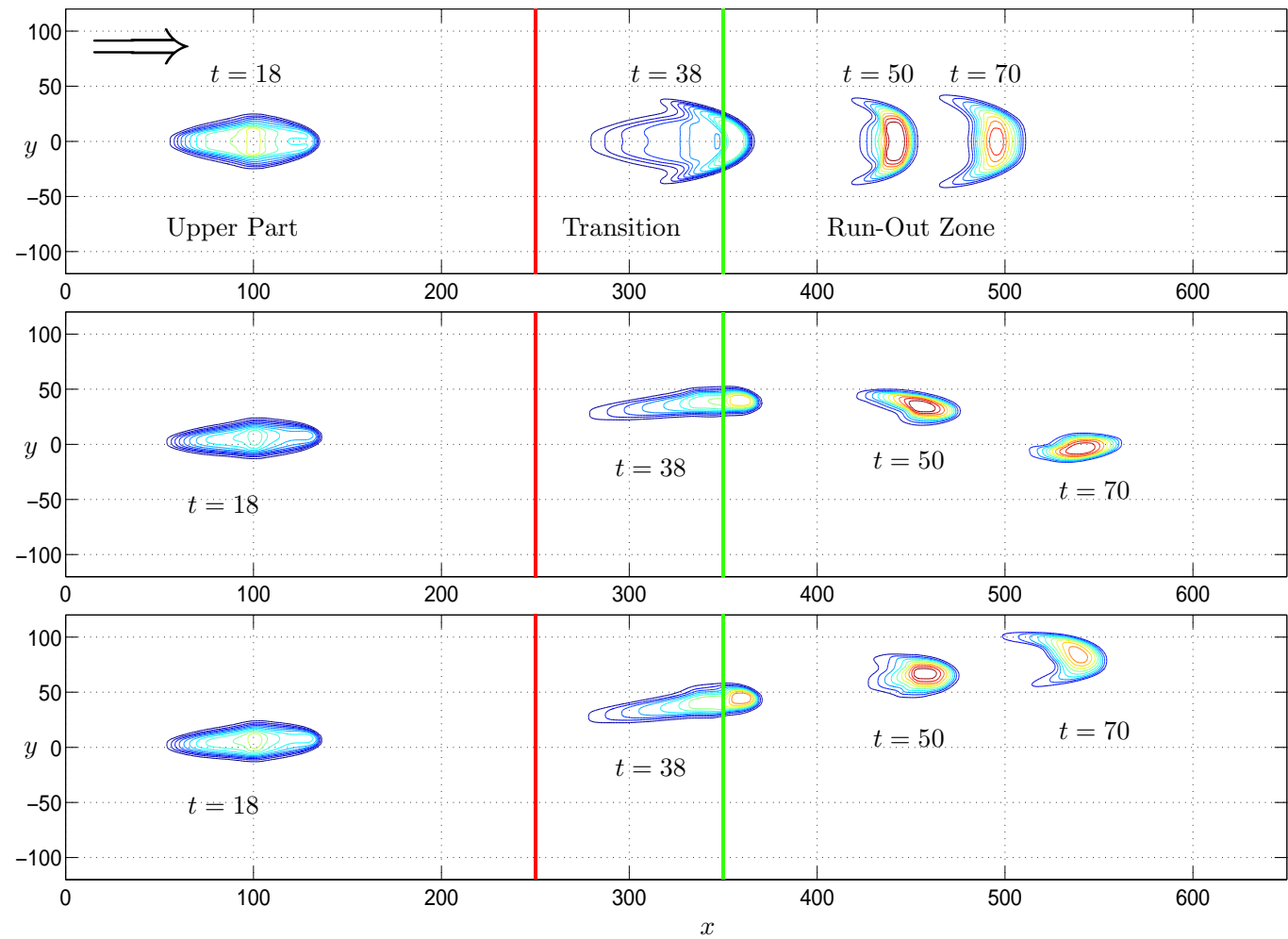

Fig. 8. Contour plots of avalanching debris flowing down curved and twisted channels: granular-fluid mixture with variable pore fluid pressure that varies (increases) linearly from the front to the tail side of the debris body. Top, middle and bottom panels correspond to bottom panels of Figs. 3, 5 and 7, respectively, that were plotted for constant pore fluid pressure throughout the body. The upper part of the chute $(x<250)$ is inclined at an angle of $45^{\circ}$, the middle part is a transition zone $(250<x<350)$ and the final part $(x>350)$ is the run-out zone. Time slices: $t=18,38,50,70$. The mass is initially kept in a hemi-spherical cap of radius 6.5 centred at $(23,0)$ and initial velocity is zero. Internal and bed friction angles of the grains are $33^{\circ}$ and $27^{\circ}$, respectively and $N_{R}=3 \times 10^{5}$.

where, $\Lambda_{f}^{\text {mean }}$ is the mean value of $\Lambda_{f}$ in the longitudinal direction between its front and rear values, $\Delta \Lambda_{f}$ the difference of $\Lambda_{f}$ at the rear end and the front of the body for the final time, $t_{0}$ the initial time and $t_{\max }$ the maximum time for numerical computation. For the simulation we take $\Lambda_{f}^{\text {mean }}=0.3$ and $\Delta \Lambda_{f}=0.3$. So, $\Lambda_{f}$ is a bilinear function of space and time with its largest value at the rear and smallest value at the front of the debris body. When $t=t_{\max }$ the maximum value of $\Lambda_{f}$ at the rear end is $50 \%$ larger (i.e., 0.45 ), and at the front $50 \%$ smaller (i.e., 0.15 ) than its value at the center (i.e., 0.3) of the body. Equation (54) thus describes a simple mechanism for the diffusion of the pore fluid pressure from the rear to the front of the debris body.

Effects of variable pore fluid pressure. Figure 8 depicts three panels for the debris flow simulation over the simply curved chute (top), the curved and twisted channel with uniform cross-section from initiation to the deposit (middle), and the curved and twisted channel with open flat run-out zone (bottom), corresponding to the last panels of Figs. 3, 5 and 7 , respectively. In these simulations the other parameters are as before, but with variable pore pressure distribution as parameterised by (54). Compared with their previous counterparts, one observes two significant influences of the variable pore pressure at the bed, $(i)$ in the forms of the debris flow surges, and (ii) run-out distances, mainly in the runout zones. In each case, it is seen that the fronts move a bit slower and the rears move faster than those for constant pore pressure distribution. The reason for this is the decreasing bed friction angle as one moves towards the tail of the body from its front. Another interesting effect is seen around the front of the body, where the surface gradient increases with increasing time and increasing value of the fluid component (compare the graphs in all three panels for $t \geq 38$ ). Similarly, the travel distances of debris bodies are also shorter than before. These phenomena can be explained somehow with reference to observations. Measurements at the base of experimental flows show that coarse-grained surge fronts have little or no pore fluid pressure. In contrast, the finer-grained thoroughly saturated debris behind surge fronts is highly liquefied by high pore pressure (Iverson and Denlinger, 2001). As we will see, the present model, when coupled with a reasonable pore pressure distribution which may be determined by an appropriately postulated equation, is able to address these problems.

\subsection{Comparison with experiments}

In this subsection we will present comparisons of simulation results with our model equations with two types of laboratory 

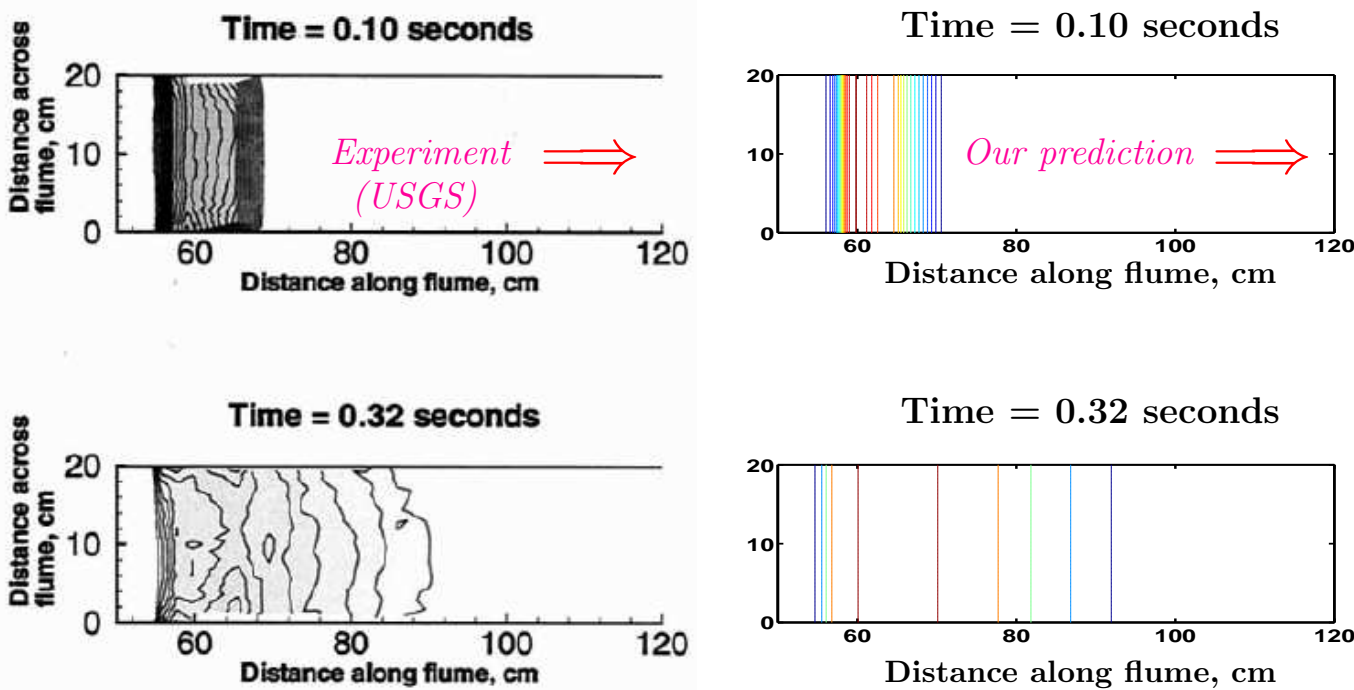

Time $=0.32$ seconds
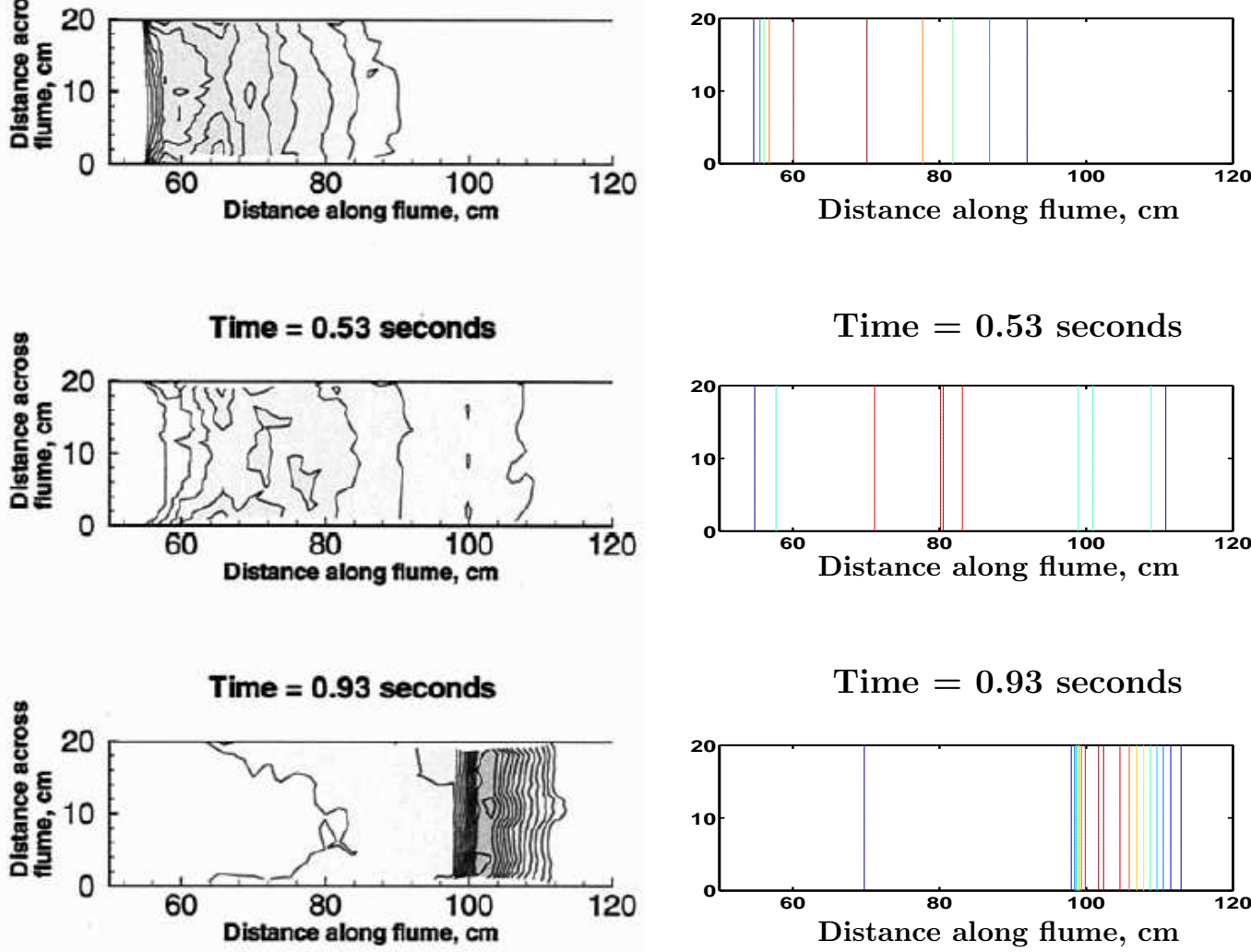

Time $=0.93$ seconds
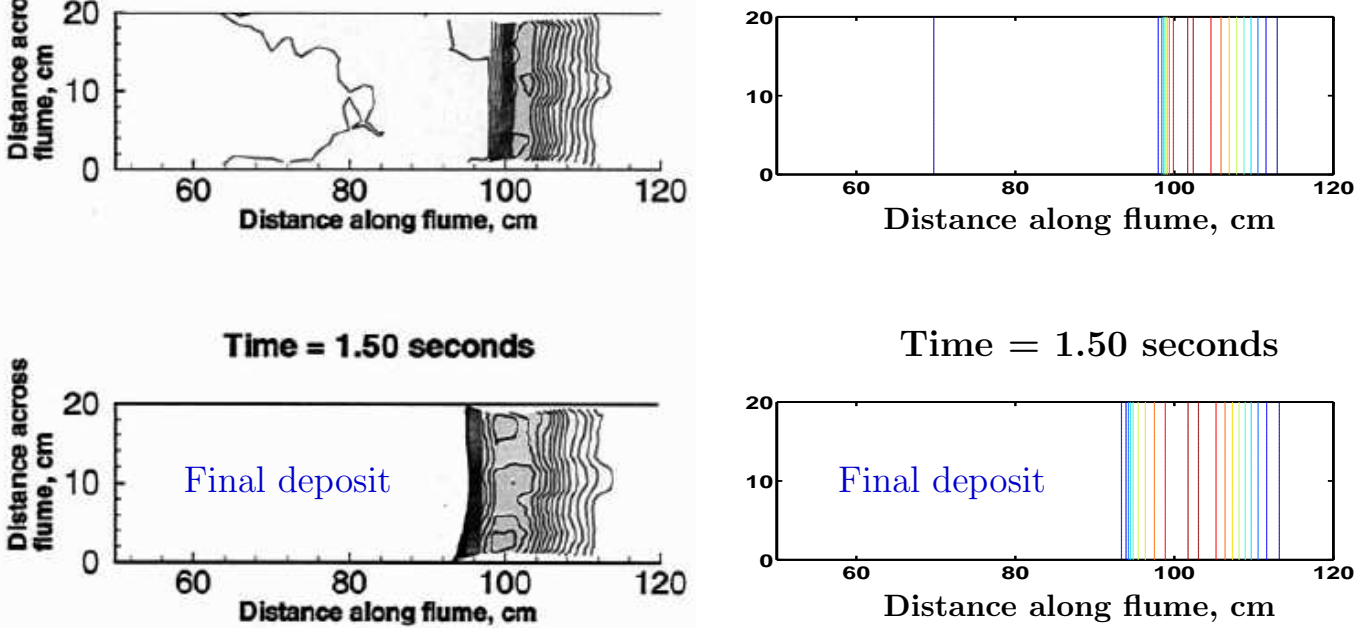

Time $=1.50$ seconds

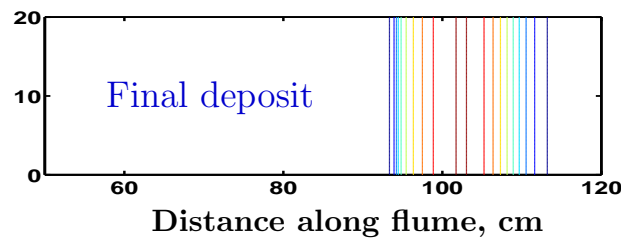

Fig. 9. Comparison between experimental data and model predictions for flow of dry sand released instantaneously from behind the gate of a $20 \mathrm{~cm}$ wide rectangular flume (USGS), left panels data from Denlinger and Iverson (2001), right panels predicted results from our model. The gate was opened at time zero. Contours are plotted normal to the bed with $1 \mathrm{~mm}$ isopaches of sand thickness for both right and left panels.

and out-door flume experiments for dry granular and debris flow, respectively. For this purpose, all data and scales are presented in dimensional units.

\subsubsection{Dry granular flow}

In this paper we compare simulation results of our equations with the data of a simple flume experiment of Denlinger and Iverson (2001). The reason is that we want to compare our 


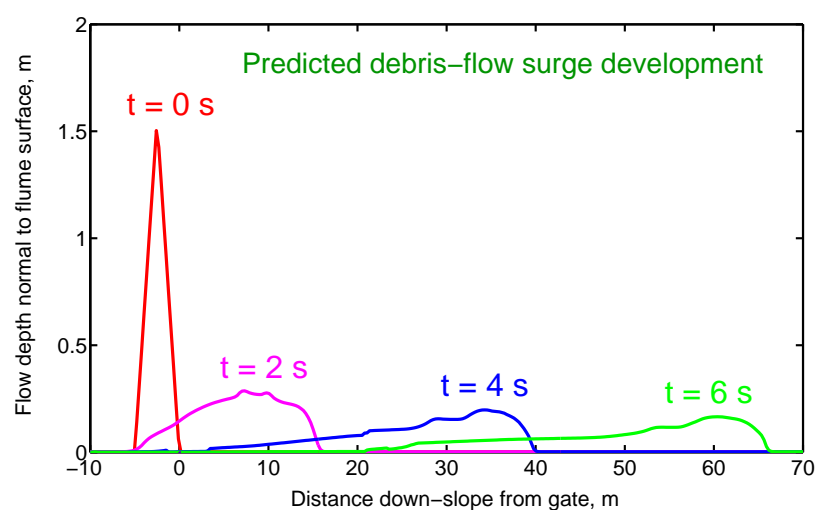

Fig. 10. Predicted profiles of debris flow surges along the longitudinal section at four successive times on an inclined rectangular flume with inclination angle $31.4^{\circ}$.

computational findings with their results and some other numerical schemes which also employed their data.

Consider the small-scale granular avalanche experiment of Denlinger and Iverson (2001) over a $20 \mathrm{~cm}$ wide, $5 \mathrm{~cm}$ high and $120 \mathrm{~cm}$ long rectangular flume inclined at an angle $31.4^{\circ}$. The flume merges continuously to the horizontal run-out and deposition zone. A volume of $290 \mathrm{~cm}^{3}$ of dry quartz sand was used in the experiment with internal and bed friction angles, $40^{\circ}$ and $29^{\circ}$, respectively. Except for small boundary layer effects, due to the initial inflow condition and confining parallel vertical walls, the flow mainly is unaffected by these boundary layers and takes place in a vertical plane parallel to these vertical walls. This means that the depth integrated deformation can be assumed to be one-dimensional along the slope direction.

Figure 9 represents a comparison between the experimental (left panels) and our simulation results (right panels) of the avalanche height, respectively, with $1 \mathrm{~mm}$ contour isopaches plotted normal to the bed surface. Comparison is made at five time slices which include the entire avalanche motion. Most important aspects of model performance can be seen while comparing the panels on the left and right columns. It took $1.5 \mathrm{~s}$ for the mass to complete deposition in both cases. Predicted timing, depth, geometry, front and tail positions, and final deposit of granular sand flow fit almost accurately with measured data of Denlinger and Iverson (2001). Some boundary layer effects, mainly in the tail side of the debris body, can be seen at time $0.93 \mathrm{~s}$ in the experimental result. Otherwise, this effect is negligible. Shocks are formed at the tail side of the body in the deposits as seen at times $0.93 \mathrm{~s}$ and $1.50 \mathrm{~s}$ in the experimental panels. They are accurately predicted by our model simulations. Qualitatively, similar results were also obtained by Denlinger and Iverson (2001). However, the overall dynamics predicted by our model simulations are obviously more accurate than those of Vollmöller (2004) in which it took a relatively large time $(2.0 \mathrm{~s})$ for the mass to come to stand still, the mass travelled significantly farther in the deposition zone, also dif- fused upslope; and the sharp gradient in the height seen in last two panels, both in the experiment and in our model performance, could not be resolved in Vollmöller's simulation.

\subsubsection{Debris flow surges and hydrographs}

\section{Advection-diffusion equation}

In order to compare numerical simulations of our model equations for debris flow with flume experiments we first need to present a short discussion on the advection-diffusion equation that will be used to determine the fluid pressure at the bed, $p^{b}(x, y, t)$. Assuming the dominant role of the pore fluid pressure distribution in the dynamics of watersaturated debris flow it is important to incorporate an evolution equation for the pore fluid pressure into the system of balance Eqs. (39)-(47). Following Iverson and Denlinger (2001), we use a simple approach in connection with the momentum equations. These authors argue that basal fluid pressure advects only passively along the flow directions, $x$ and $y$, and that it also diffuses simultaneously in the normal, $z$, direction leading to the advection-diffusion equation $\partial p^{b} / \partial t+u \partial p^{b} / \partial x+v \partial p^{b} / \partial y=\left.D\left(\partial^{2} p / \partial z^{2}\right)\right|_{b e d}$, where $D$ is the pore pressure diffusivity in the mixture. We will employ this equation in our simulation with $D=10^{-4}$ as employed by Iverson and Denlinger (2001).

\section{Comparison of the model with flume experiments}

Next, we consider a relatively large debris flow flume experiment with about $10 \mathrm{~m}^{3}$ of water-saturated sand and gravel. The rectangular flume which is inclined at angle $31.4^{\circ}$ is $2 \mathrm{~m}$ wide and $95 \mathrm{~m}$ long and is connected with an additional flat run-out surface with slope $2.5^{\circ}$. The flume geometry, initial conditions and parameter values are taken from Denlinger and Iverson (2001).

Debris flow surges: Figure 10 depicts the evolution of the speed and shape of the debris flow surge after its release from the gate. Predicted results indicate that a blunt snout is quickly developed and is only modestly changed as the debris front advanced farther downslope. The snout is always accelerating on the uniform slope, but the liquefied tail behind the snout is highly elongated and accelerated less rapidly than the snout itself. It was the reason for the surge shape to stretch in length and decrease in height with time and travel distance. These phenomena are quite common in nature and in experiments. These results are similar to those presented in Iverson (1997) but more convincing than those presented in Savage and Iverson (2003). Alternatively, simulations with no pore pressure (i.e., dry granular flow) would produce only a finely tapered (smooth) leading edge.

Debris flow hydrographs: Figure 11 presents a test of the model simulations against the measured debris flow hydrograph (i.e., the flow depth as a function of time at fixed location). We assume that the flow is uniform in the cross-section in the inclined portion of the flume. The left panels in Fig. 11 

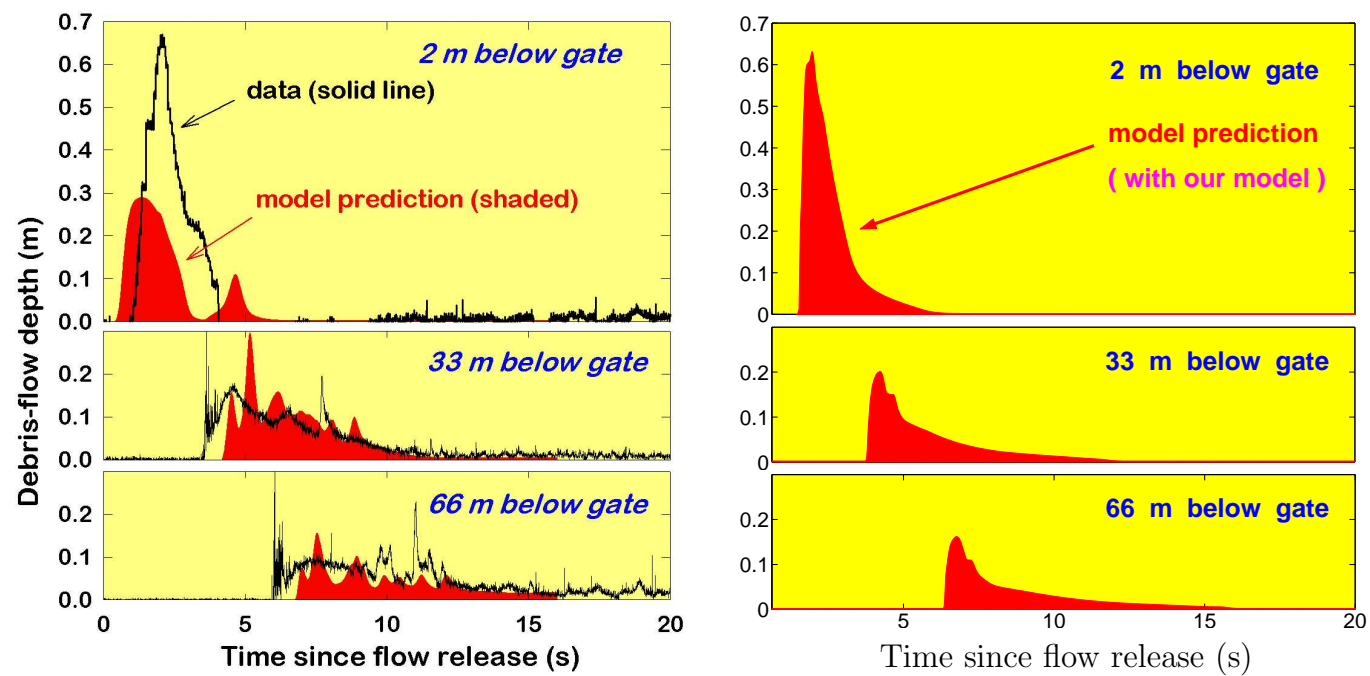

Fig. 11. Comparison between measurements and two model predictions. Left: Experimental data of flow depth at three cross sections of a water-saturated debris flow at USGS flume, 24 July, 1995 and numerical results predicted by Denlinger and Iverson (2001). Right: Numerical results predicted by our model.

compare time series of measured depths at three downstream locations with the simulated results of Denlinger and Iverson (2001), whilst the right panels are results from our model simulation. Comparison of the data and model predictions reveal that both models predict the speed of the debris flow surge reasonably well, and rather well with our simulation. In the Denlinger and Iverson (2001) model simulation the most significant prediction error occurred for the location at $2 \mathrm{~m}$ down-slope from the gate. Their model predictions are getting better farther down-slope. Our model simulation can more convincingly predict the attenuation of the surge front, but does not show any backup of the surge into multiple surges, that can be seen in their simulation. Reliable simulation of the instability that causes a single surge to develop into multiple surges is still poorly understood, it requires more accurate knowledge of physical instabilities. In both simulations, as debris flow surges (fronts) reach crosssections $33 \mathrm{~m}$ and $66 \mathrm{~m}$ from the gate their "primary waveforms" have markedly accelerated, elongated and attenuated.

\section{Discussion and conclusions}

In this paper a reduced two-phase solid-fluid model was presented that is capable to describe the dynamics of the catastrophic motion of water saturated soil down arbitrary channelised topographies from initiation to run-out. The basic equations are those of classical binary mixture, however with the simplifying first assumption imposed that the fluid constituent velocity differs only by a negligible amount from the constituent velocity of the solids phase. It is paired with a second ad-hoc assumption on $\Lambda_{f}$ which is of constitutive nature explicitly given by us algebraically. Both assumptions are essentially due to Iverson (1997) and are the strength and weakness of the model. The latter assumption, paired with the supposition that the pore space suffers only negligible variations, allowed the drastic simplification to reduce the binary mixture model equations to alternative balance equations with one-constituent properties. Mass balance of this reduced continuum requires the solids velocity field to be solenoidal, and the momentum balance equation takes its common one-constituent form with a Cauchy stress tensor that is the sum of the solids stress and the interstitial fluids stress. The interaction force disappears as a dynamical quantity from this reduced formulation. Closure conditions are therefore only needed for the two peculiar stresses, and we proposed a cohesionless Coulomb type dry friction law for the solids stress and a Newtonian viscous type postulate for the interstitial fluid. The equations differ from the earlier equations of the dynamics of dry granular avalanches exhibiting Coulomb type friction only by the fluid presence and the viscous fluid deviator stress.

The non-dimensionalisation of the equations and the derivation of the reduced depth-integrated two-dimensional final equations are based on the introduction of an orthogonal curvilinear coordinate system following the thalweg of the channel and polar coordinates in the cross sections perpendicular to it, and the geometric assumption that the moving masses are thin and long. The corresponding asymptotic analysis has not been repeated because it would reproduce earlier computations done for the extended SavageHutter model (see Pudasaini and Hutter, 2003). We restricted this analysis only to those terms which are new and are stated in (43)-(47). For vanishing fluid contribution $\left(\Lambda_{f}=0, N_{R} \rightarrow \infty\right)$ and torsion free bed topography $(\eta=1)$ our new model equations exactly degenerate to the previous model equations of Savage-Hutter-type (e.g., Savage and Hutter, 1989, 1991; Gray et al., 1999) which was not possible by Iverson and Denlinger (2001) model, which in fact, aimed to generalise the Savage-Hutter model. 
In this process it turned out that the component of the momentum balance perpendicular to the basal surface reduced to a balance of normal fluid-pressure gradient, normal solid stress gradient, gravity force and "centrifugal" force components (see Eq. 27). This required an additional closure relation that would not be needed in a full theory, namely a postulate how the gravity plus "centrifugal" force component would be split among the fluid pressure and the solid normal stress perpendicular to the base. An additive decomposition was suggested assigning the $\Lambda_{f}$ part to the fluid pressure and $\left(1-\Lambda_{f}\right)$ part to the solid normal stress. This division is the weakness of this formulation, because it is ad-hoc, but the introduced split is a common assumption in soil physics where the normal fluid-pressure, $p$, is divided among the constituents according to some proportion, (see, Iverson and Denlinger, 2001; De Boer, 2000; Ehlers et al., 2004; Vulliet and Laloui, 2001; dell'Isola, 1998). Indeed, it is dynamically important: because without this assumption the model equations cannot be closed as a set of depth integrated equations. The separation parameter $\Lambda_{f}$ enters as a new field variable of the depth integrated equations, for which a phenomenological closure condition must be postulated. This does not make the model very appealing and succeptable to poor constitutive guessing. Nevertheless, the computational results performed for avalanching debris flows down curved and twisted channels, show a drastic influence of the fluid pressure via the parameter $\Lambda_{f}$. For large fluid pressure, i.e., large values of $\Lambda_{f}$, the travelled distances of the debris masses are considerably larger than for small values. It is interesting to note and physically due to the fact that the fluid pressure in the interior of the bodies is larger than at the unconstrained boundaries. The geometries of the deposits of these flows therefore show Barchan type forms when channel compactions to the sides are absent but otherwise do not.

Since $\Lambda_{f}$ has evidently been shown to be the decisive parameter it is important that this parameter is given attention for its proper parameterisation. To assign to it only a constant value is too rough to generate reliable results. We have therefore also provided suggestions for its evolution as in Iverson and Denlinger (2001) and Savage and Iverson (2003) but have postulated a simple bilinear function in this paper.

More importantly, we have also compared numerical simulations of our model equations with a small-scale laboratory dry granular flow and a large-scale water-saturated debris flow over rectangular flumes by employing an advection-diffusion equation for the determination of the pore fluid pressure distribution along the avalanching debris flow (Iverson, 1997; Denlinger and Iverson, 2001; Savage and Iverson, 2003). Very good agreement between the theory and experiments is established.

Acknowledgements. We would like to thank an anonymous referee for his careful review that improved the paper considerably. Thanks are also due to R. M. Iverson for providing us with experimental figures.
Edited by: G. Wieczorek

Reviewed by: one referee

\section{References}

de Boer, R.: Theory of Porous Media: Highlights in Historical Development and Current State, Springer, 2000.

dell'Isola, F. and Hutter, K.: A qualitative analysis of the dynamics of a sheared and pressurized layer of saturated soil, Proc. R. Soc. A, 454, 3105-3120, 1998.

Denlinger, R. P. and Iverson, R. M.: Flow of variably fluidised granular masses across three-dimensional terrain. 2. Numerical predictions and experimental tests, J. Geophys. Res., 106, 552-566, 2001.

Denlinger, R. P. and Iverson, R. M.: Granular avalanches across irregular three-dimensional terrain: 1. Theory and computation, J. Geophys. Res., 109(F1), art. no. F01014 MAR 27, 2004.

Ehlers, W., Graf, T., and Ammann, M.: Deformation and Localization Analysis of Partially Saturated Soil, Comput. Meth. Appl. Mech. Eng., 193, 2885-2910, 2004.

Gray, J. M. N. T., Wieland, M., and Hutter, K.: Gravity-driven free surface flow of granular avalanches over complex basal topography, Proc. R. Soc. A, 455, 1841-1874, 1999.

Harten, A.: High resolution schemes for hyperbolic conservation laws, J. Comput. Phys., 49, 357-393, 1983.

Harten, A., Osher, S. Engquist, B., and Chakravarthy, R.: Some results on uniformly high-order accurate essentially nonoscillatory schemes, Appl. Num. Math., 2, 347-377, 1986.

Hubbert, M. K. and Rubey, W. W.: Role of fluid pressure in mechanics of overthrust faulting, Geol. Soc. America, Bulletin, 70, 115-166, 1959.

Hungr, O.: A model for the runout analysis of rapid flow slides, debris flows, and avalanches, Can. Geotech. J., 32, 610-623, 1995.

Hutter, K., Wang, Y., and Pudasaini, S. P.: The Savage-Hutter avalanche model. How far can it be pushed?, Phil. Trans. R. Soc. A, 363(1832), 1507-1528, 2005.

Iverson, R. M.: The Physics of Debris Flows, Rev. Geophys., 35, 245-296, 1997.

Iverson, R. M. and Denlinger, R. P.: Flow of variably fluidised granular masses across three-dimensional terrain: 1. Coulomb mixture theory, J. Geophys. Res., 106, 537-552, 2001.

Iverson, R. M., Logan, M., and Denlinger, R. P. Granular avalanches across irregular three-dimensional terrain: 2. Experimental tests, J. Geophys. Res., 109(F1), art. no. F01015 MAR 27, 2004.

Jiang, G. S. and Tadmor, E.: Nonoscillatory central schemes for multidimensional hyperbolic conservation laws, SIAM J. Sci. Comput., 19, 1892-1917, 1998.

Koschdon, K. and Schäfer, M.: A Lagrangian-Eulerian finitevolume method for simulating free surface flows of granular avalanches, in: Dynamic Response of Granular and Porous Materials under Large and Catastrophic Deformation, edited by: Hutter, K., Kirchner, N., Lecture Notes in Applied and Computational Mechanics, 11, 83-108, Springer, Berlin Heidelberg New York, 2003.

Kröner, D.: Numerical schemes for conservation laws, B. G. Teubner Stuttgart, 1997.

LeVeque, R. J.: Numerical methods for conservation laws, Birkhäuser, Basel, Boston, New York, 1990.

Nessyahu, H. and Tadmor. E.: Non-oscillatory central differencing for hyperbolic conservation laws, J. Comput. Phys., 87, 408-463, 1990. 
Patra, A. K., Bauer, A. C., Nichita, C. C., Pitman, E. B., Sheridan, M. F., Bursik, M, Rupp, B., Weber, A., Stinto, A., Namikawa, L., and Renschler, C.: Parallel Adaptive Numerical Simulation of Day Avalanches over Natural Terrain, J. Volcanology Geotherm. Res., 139(1-2), 1-21, 2005.

Pitman, E. B., Nichita, C. C., Patra, A. K., Bauer, A. C., Bursik, M., and Weber, A.: A Model of Granular Flows over an Erodible Surface, Discrete Contin. Dynam. Syst. B, 3, 589-599, 2003.

Pitman, E. B. and Le, L.: A two-fluid model for avalanche and debris flows, Phil. Trans. R. Soc. A, 363(1832), 1573-1601, 2005.

Pudasaini, S. P.: Dynamics of Flow Avalanches Over Curved and Twisted Channels: Theory, Numerics and Experimental Validation, PhD Dissertation, Darmstadt University of Technology, Germany, 2003.

Pudasaini, S. P. and Hutter, K.: Rapid Shear Flows of Dry Granular Masses Down Curved and Twisted Channels, J. Fluid Mech., 495, 193-208, 2003.

Pudasaini, S. P. and Hutter, K.: Granular Avalanche Model in Arbitrarily Curved and Twisted Mountain Terrain: a Basis for the Extension to Debris Flow, in: Debris-Flow Hazards Mitigation: Mechanics, Prediction and Assessment, edited by: Rickenmann, D. and Chen, C.-L., Millpress, 1, 491-502, 2003a.

Pudasaini, S. P., Eckart, W., and Hutter, K.: Gravity-Driven Rapid Shear Flows of Dry Granular Masses in Helically Curved and Twisted Channels, Math. Models Meth. Appl. Sci., 13(7), 10191052, 2003.

Pudasaini, S. P., Wang, Y., and Hutter, K.: Rapid motions of freesurface avalanches down curved and twisted channels and their numerical simulations, Phil. Trans. R. Soc. A, 363(1832), 15511571, 2005a.

Pudasaini, S. P., Hsiau, S.-S., Wang, Y., and Hutter, K.: Velocity measurements in dry granular avalanches using particle image velocimetry technique and comparison with theoretical predictions, Phys. Fluids, 17(9), art. no. 093301 SEP, 2005 b.

Savage, S. B. and Hutter, K.: The motion of a finite mass of granular material down a rough incline, J. Fluid Mech., 199, 177-215, 1989.
Savage, S. B. and Hutter, K.: Dynamics of avalanches of granular materials from initiation to runout, Part I: Analysis, Acta Mech., 86, 201-223, 1991.

Savage, S. B. and Iverson, R. M.: Surge dynamics coupled to porepressure evolution in debris flow, in: Debris-Flow Hazards Mitigation: Mechanics, Prediction and Assessment, edited by: Rickenmann, D. and Chen, C.-L., Millpress, 1, 503-514, 2003.

Tai, Y. C., Noelle, S., Gray, J. M. N. T., and Hutter, K.: Shockcapturing and front-tracking methods for granular avalanches, J. Comput. Phys., 175, 269-301, 2002.

Takahashi, T.: Debris flow, In IAHR-AIRH Monograph Series A, Balkema, 1991.

Toro, E. F.: Shock-Capturing Methods for Free-Surface Shallow Flows, John Wiley \& Sons Ltd., 2001.

Vollmöller, P.: A shock-capturing wave-propagation method for dry and saturated granular flows, J. Comput. Phys., 199, 150-174, 2004.

Vulliet, L. and Laloui, L.: Mechanics of multiphase porous media-application to unsaturated soils, in: Continuum mechanics and applications in geophysics and environment, edited by: Straughan. B., Greve, R., Ehrentraut, H. and Wang, Y. Springer, Berlin, pp. 153-180, 2001.

Wang, Y., Hutter, K., and Pudasaini, S. P.: The Savage-Hutter Theory: a System of Partial Differential Equations for Avalanche Flows of Snow, Debris and Mud, J. App. Math. Mech., 84(8), 507-527, 2004.

Yee, H. C.: Construction of explicit and implicit symmetric TVD schemes and their applications, J. Comput. Phys., 68, 151-179, 1987.

Zwinger, T., Kluwick, A., and Sampl, P.: Numerical Simulation of Dry-Snow Avalanche Flow over Natural Terrain, in: Lecture Notes in Applied and Computational Mechanics - LNACM, Dynamic Response of Granular and Porous Materials under Large and Catastrophic Deformations, edited by: Hutter, K. and Kirchner, N., Springer Verlag, 11, 161-194, 2003. 